\title{
Quantifying interindividual variability and asymmetry of face-selective regions: A probabilistic functional atlas
}

\author{
Zonglei Zhen ${ }^{\mathrm{a}, \mathrm{c}, 1}$, Zetian Yang ${ }^{\mathrm{a}, \mathrm{c}, 1}$, Lijie Huang ${ }^{\mathrm{a}, \mathrm{c}}$, Xiang-zhen Kong ${ }^{\mathrm{a}, \mathrm{c}}$, Xu Wang ${ }^{\mathrm{a}, \mathrm{c}}$, Xiaobin Dang ${ }^{\mathrm{a}, \mathrm{c}}$, \\ Yangyue Huang a,c, Yiying Song ${ }^{\mathrm{a}, \mathrm{c}}$, Jia Liu ${ }^{\mathrm{b}, \mathrm{c}, *}$
}

a State Key Laboratory of Cognitive Neuroscience and Learning E' IDG/McGovern Institute for Brain Research, Beijing Normal University, Beijing 100875, China

b School of Psychology, Beijing Normal University, Beijing 100875, China

c Center for Collaboration and Innovation in Brain and Learning Sciences, Beijing Normal University, Beijing 100875, China

\section{A R T I C L E I N F O}

\section{Article history:}

Received 8 October 2014

Accepted 5 March 2015

Available online 13 March 2015

\section{Keywords:}

Fusiform face area (FFA)

Occipital face area (OFA)

Superior temporal sulcus (STS)

Interindividual variability

Asymmetry

\begin{abstract}
A B S T R A C T
Face-selective regions (FSRs) are among the most widely studied functional regions in the human brain. However, individual variability of the FSRs has not been well quantified. Here we use functional magnetic resonance imaging ( $\mathrm{fMRI}$ ) to localize the FSRs and quantify their spatial and functional variabilities in 202 healthy adults. The occipital face area (OFA), posterior and anterior fusiform face areas (pFFA and aFFA), posterior continuation of the superior temporal sulcus (pcSTS), and posterior and anterior STS (pSTS and aSTS) were delineated for each individual with a semi-automated procedure. A probabilistic atlas was constructed to characterize their interindividual variability, revealing that the FSRs were highly variable in location and extent across subjects. The variability of FSRs was further quantified on both functional (i.e., face selectivity) and spatial (i.e., volume, location of peak activation, and anatomical location) features. Considerable interindividual variability and rightward asymmetry were found in all FSRs on these features. Taken together, our work presents the first effort to characterize comprehensively the variability of FSRs in a large sample of healthy subjects, and invites future work on the origin of the variability and its relation to individual differences in behavioral performance. Moreover, the probabilistic functional atlas will provide an adequate spatial reference for mapping the face network.
\end{abstract}

(c) 2015 Elsevier Inc. All rights reserved.

\section{Introduction}

People show considerable interindividual variability across physical characteristics: from mass and height to the placement of facial features and visceral organs. In particular, the human brain is characterized by striking interindividual variability in structure and function (Frost and Goebel, 2012; Hasnain et al., 1998; Sugiura et al., 2007; Van Essen and Dierker, 2007; Xiong et al., 2000; Zilles and Amunts, 2013). In the past two decades, dramatic interindividual variability has been observed in both sulcal/gyral patterns and anatomical features (e.g. cortical thickness, volume, and shape) at the macroscopic level (Bartley et al., 1997; Fischl and Dale, 2000; Fischl et al., 2008; Thompson et al., 1996, 1998; Zilles et al., 2013) and in the size and extent of cytoarchitectonic areas defined at the microscopic level (Amunts et al., 1999; Caspers et al., 2006; Rademacher et al., 2001; Scheperjans et al., 2007; Zilles and Amunts, 2010). Recently, significant variability has also been revealed in anatomical and functional connectivity of the brain

\footnotetext{
* Corresponding author at: Room 422, Yingdong Building, 19 Xinjiekouwai St, Haidian District, Beijing 100875, China. Fax: + 861058806154.

E-mail address: liujia@bnu.edu.cn (J. Liu).

1 These authors contributed equally to this work.
}

(Burgel et al., 2006; Mueller et al., 2013). This variability provides vital information on our understanding of brain organization and the neural basis of human behavior and cognition (Kanai and Rees, 2011; Zilles and Amunts, 2013). In contrast to the large amount of work being devoted to assessing structural and connectional variability in the brain, individual variability in functionally defined regions (i.e., homogenous regions with specific response properties) is rarely quantified (Frost and Goebel, 2012; Hasnain et al., 1998; Nieto-Castanon and Fedorenko, 2012; Saxe et al., 2006; Van Horn et al., 2008). Here, we used functional magnetic resonance imaging (fMRI) to investigate the variability of faceselective regions (FSRs), which are selectively activated by faces versus non-face objects.

Face recognition is a highly developed skill in humans. A single glance at a face provides rich information on identity, gender, expression, age, and mood. Substantial evidence demonstrates that a dedicated network of the FSRs is recruited for face processing (Haxby et al., 2000; Ishai, 2008; Rossion et al., 2012; Zhen et al., 2013; Zhu et al., 2011). These FSRs could be reliably localized across individuals even with a short period of functional localizer scans (Fox et al., 2009; Kawabata Duncan and Devlin, 2011). Among them, the most reliable regions are located in the occipitotemporal cortex. Specifically, one FSR is in the inferior occipital gyrus (IOG, or occipital face area, OFA) 
(Gauthier et al., 2000; Liu et al., 2010); three FSRs are in the fusiform gyrus (FG, or fusiform face area, FFA), located in the posterior, middle and anterior parts of the FG (i.e., pFFA, mFFA, and aFFA) (Engell and McCarthy, 2013; Gauthier et al., 1999; Kanwisher et al., 1997; Nestor et al., 2011; Weiner and Grill-Spector, 2010), and three FSRs are in the superior temporal sulcus (STS), located at the posterior continuation of the STS, the posterior STS, and the anterior STS (i.e., pcSTS, pSTS, and aSTS) (Pinsk et al., 2009; Pitcher et al., 2011a; Puce et al., 1998). These regions are thought to process different aspects of faces (Haxby et al., 2000): the region located in the IOG is involved in early perception of facial features; those regions located in the FG analyze the invariant aspects of faces that underlie recognition of individuals (Kanwisher et al., 1997; McCarthy et al., 1997; Pitcher et al., 2011b) and those regions located in the STS process the changeable aspects of faces such as expressions (Phillips et al., 1997), direction of eye gaze, and lip movements, for facilitating social communication (Allison et al., 2000; Hoffman and Haxby, 2000; Lahnakoski et al., 2012; Puce et al., 1998).

Although great advances have been made in recent years in applying fMRI to characterize these FSRs, previous studies have mostly focused on common properties of the FSRs across individuals. The interindividual variability of the FSRs is rarely investigated, although the variability has commonly been observed in previous studies (Fox et al., 2009; Goffaux et al., 2011; Kanwisher et al., 1997; Pinsk et al., 2009). Only recently have four groups begun to develop probabilistic atlases (or maps) to characterize the variability of face-selective activation (Engell and McCarthy, 2013; Frost and Goebel, 2012; Julian et al., 2012; Tahmasebi et al., 2012). Three of them (Engell and McCarthy, 2013; Julian et al., 2012; Tahmasebi et al., 2012) constructed the atlases at the voxel level, not at the FSR level; therefore, they didn't characterize the interindividual variability of the FSRs, which is critical information for understanding the structural-functional correspondence in the brain. One study indeed created the atlas at the FSR level (Frost and Goebel, 2012), but it is only based on 10 subjects and one FSR (i.e., the FFA). Therefore, it is unclear how representative the atlas is and what the spatial relation among the FSRs is (e.g., to what extend does the FFA overlap with the OFA?). The present study extended the previous studies by identifying six well-studied FSRs in each individual of a large cohort of subjects, and then constructing a probabilistic atlas of the FSRs to characterize its spatial and functional variabilities.

Specifically, to capture reliably the variability of the FSRs, 202 subjects were scanned with a robust dynamic face localizer (Fox et al., 2009; Pitcher et al., 2011a) in the same scanner and with the same MR protocol. First, the six well-studied FSRs, the OFA, pFFA, aFFA, pcSTS, pSTS, and aSTS, were delineated in each individual using a semi-automated procedure. Second, a probabilistic atlas was created to quantify the spatial variability of FSRs, which contained precise stereotaxic information on both interhemispheric and interindividual differences. Third, the functional and spatial variabilities of the FSRs were characterized on four features, including face selectivity, volume, location of peak activation, and anatomical location. Besides interindividual variability, the interhemispheric variability (i.e. hemispheric asymmetry) of these features was also examined.

\section{Materials and methods}

\section{Data description}

\section{Subjects}

Two hundred and two college students ( 124 females; mean age = 20.3 years, standard deviation (SD) $=0.88$ years), from Beijing Normal University, China, participated in the study. All subjects had normal or corrected-to-normal vision. The study was approved by the Institutional Review Board of Beijing Normal University. Written informed consent was obtained from all subjects before they took part in the experiment.

\section{Localizer paradigm}

A dynamic face localizer was used to define the FSRs. Empirical evidence indicates that the task could elicit reliable activations of FSRs across subjects (Fox et al., 2009; Pitcher et al., 2011a). Specifically, the dynamic face localizer data were acquired over three blocked-design functional runs, each of which lasted $198 \mathrm{~s}$. Each run contained two block sets, intermixed with three 18-s rest blocks at the beginning, middle and end of the run. Each block set consisted of four blocks of four stimulus categories (i.e., faces, scenes, objects, and scrambled objects), with each stimulus category presented in an 18-s block. Each block contained six 3-s movie clips from an object category, which were randomly drawn from a pool of 60 clips (for more details on the stimuli, see Pitcher et al., 2011a). The order of stimulus category blocks in each run was palindromic and was randomized across runs. Subjects were instructed to view passively movie clips during scanning. In the present study, we defined the FSRs by the contrast of faces versus objects (e.g., Kanwisher et al., 1997). Other conditions in the localizer task were designed to localize the object-selective and scene-selective regions, which will be characterized in other studies.

\section{Image acquisition}

Functional and structural MR imaging was done at BNU Imaging Center for Brain Research, Beijing, China, on a Siemens 3 T wholebody scanner (MAGENTOM Trio, a Tim system) with a 12-channel phased-array head coil. Functional blood-oxygen-level-dependent (BOLD) images were acquired with a $\mathrm{T}^{*}$-weighted gradient-echo, echo-planar-imaging (GRE-EPI) sequence ( $\mathrm{TR}=2 \mathrm{~s}$, echo time $=$ $30 \mathrm{~ms}$, flip angle $=90^{\circ}$, in-plane resolution $=3.1 \times 3.1 \mathrm{~mm}$ ). Wholebrain coverage for the functional data was obtained using 30 contiguous interleaved $4.8 \mathrm{~mm}$ axial slices. Structural T1-weighted images were acquired with a 3D magnetization-prepared rapid acquisition gradient echo (MP-RAGE) sequence $(\mathrm{TR} / \mathrm{TE} / \mathrm{TI}=2530 / 3.39 / 1100 \mathrm{~ms}$, flip angle $=7^{\circ}$ ) for spatial normalization. Earplugs were used to attenuate scanner noise, and head motion was restrained with a foam pillow and extendable padded head clamps.

\section{Quality control}

Two predefined criteria were used to assess the quality of functional MR images. First, to minimize the motion artifacts during scanning, subjects with excessive motion were excluded. Functional volumes with movements exceeding $2 \mathrm{~mm}$ in translation errors or $2^{\circ}$ in rotation errors (in any direction) were flagged for excessive motion. Subjects with more than 20 flagged volumes across all runs were excluded from further analysis. Second, to minimize the error caused by misalignment of functional and anatomical volumes, subjects with large errors of registration were excluded. Specifically, the registration quality was checked visually by overlaying the normalized functional volume on the MNI152 template.

No subject showed excess head motion or visually detected registration errors; therefore, no subject was excluded, and data from all subjects were analyzed to characterize the interindividual variability of the FSRs.

\section{Image processing}

\section{Activation analysis}

Functional images were analyzed with FEAT (FMRI Expert Analysis Tool) Version 5.98, part of FSL (FMRIB's Software Library, www.fmrib. ox.ac.uk/fsl). The first-level analysis was conducted separately on each run and each session (i.e., subject). Preprocessing included the following steps: motion correction, brain extraction, spatial smoothing with a Gaussian kernel (FWHM $=6 \mathrm{~mm}$ ), grand-mean intensity normalization, and high-pass temporal filtering (120 s cutoff). Statistical analyses on time series were performed using FILM (FMRIB's Improved Linear Model) with a local autocorrelation correction. A boxcar kernel was convolved with a gamma hemodynamic response 
function, and its temporal derivative was used to model blood oxygen level-dependent (BOLD) signal changes. Six parameters from motion-correction were also included in the model as regressors of no interest to account for the effect of residual head movements. A second-level analysis was then done to combine all runs within each session. Specifically, the parameter (i.e. beta) image from the first-level analysis was first aligned to the individual's structural images using FLIRT (FMRIB's linear image registration tool) with 6 degrees of freedom, and then warped to the MNI152 template using FNIRT (FMRIB's nonlinear image registration tool) running with the default parameters. The spatially normalized parameter images (resampled to 2 -mm isotropic voxels) were then summarized across runs in each session using a fixed-effect model. The statistic images from the secondlevel analysis were then used to identify the subject-specific FSRs.

\section{Subject-specific FSR delineation}

We focused on the FSRs located in the occipitotemporal cortex, including OFA, pFFA, aFFA, pcSTS, pSTS, and aSTS. Of note, our data did not allow the differentiation of the pFus-faces and mFus-faces (Engell and McCarthy, 2013; Pinsk et al., 2009; Weiner and Grill-Spector, 2010) because most subjects showed only one cluster spanning from the posterior to middle FG. Moreover, for subjects who did show multiple clusters here, we often had trouble classifying them as either pFusfaces or mFus-face, possibly because of the low resolution of our fMRI data or the spatial smoothing used in the analysis (Weiner and GrillSpector, 2011). Another possibility is that the dynamic stimuli were not efficient for differentiating the pFus-faces and mFus-faces, as a previous study showed that fusiform face regions are more sensitive to static than to dynamic information (Pitcher et al., 2011a). Therefore,
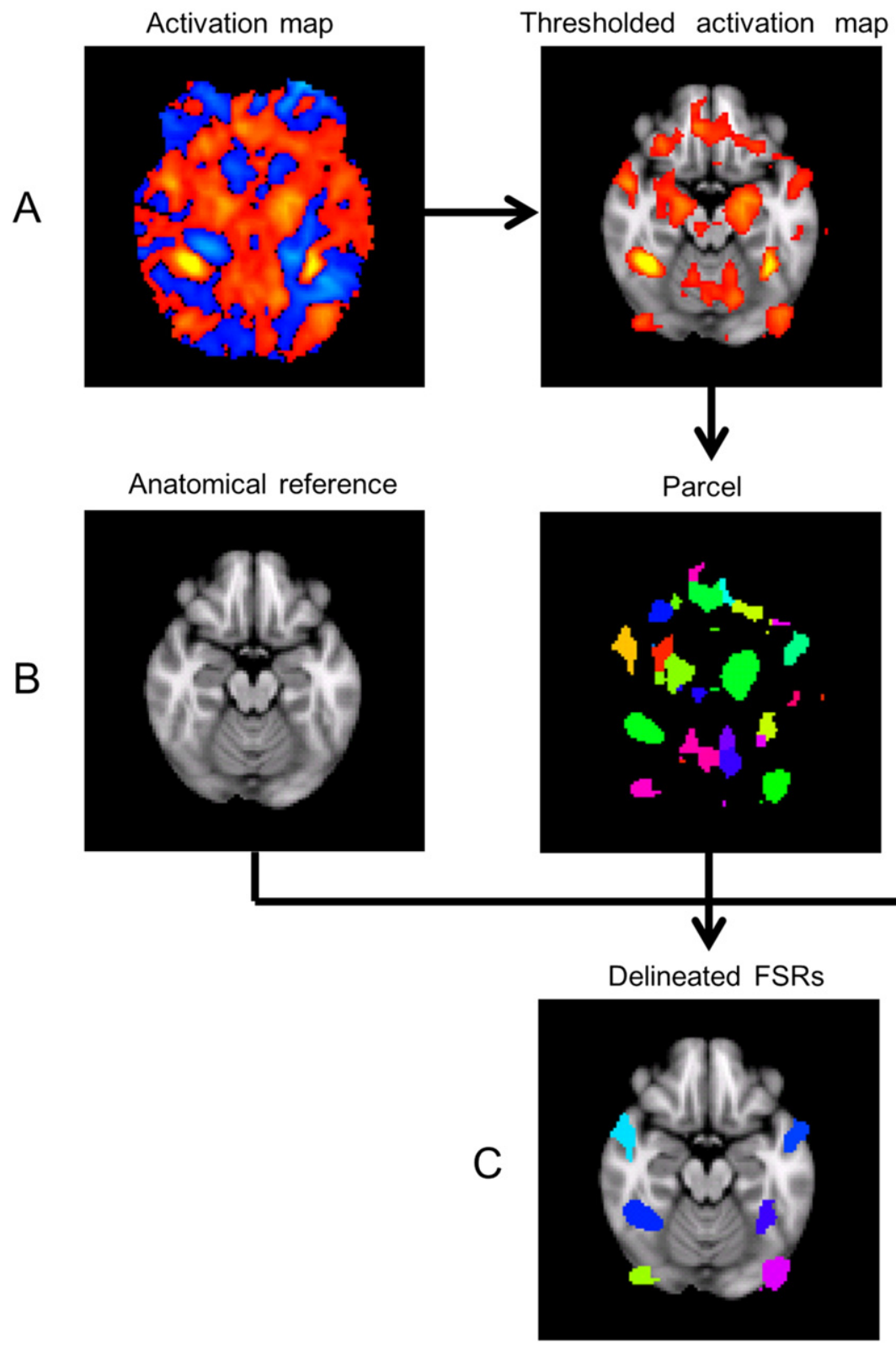

Functional reference

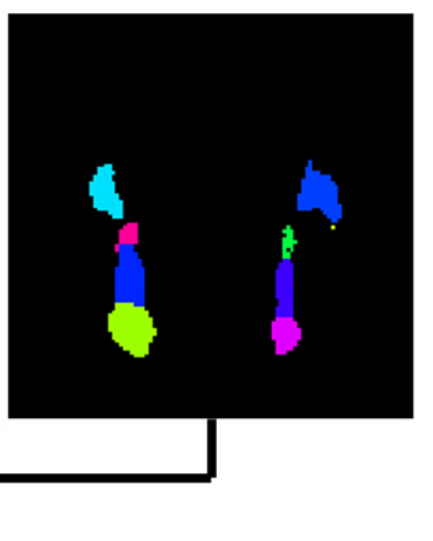

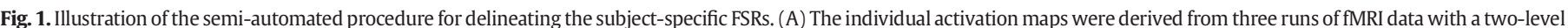

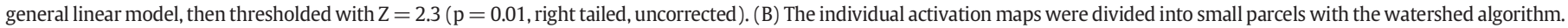

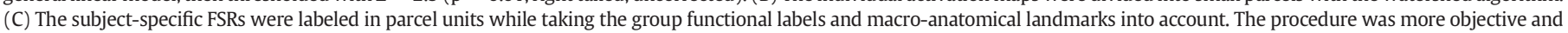
efficient than traditional labeling by voxel-wise handpicking. 
we defined the clusters that spanned from the posterior to middle FG as a single region (i.e., the pFFA).

The subject-specific FSRs were delineated by seven raters specializing in the ventral visual pathway. Three sources of maps were used in combination in the delineating procedure, namely the subject-specific activation map, an FSR spatial reference, and a macro-anatomical landmark reference. Specifically, the subject-specific activation map was the statistical images derived from the three-run fixed effect analysis; the FSR spatial reference was a map of the high-probability parcels derived from the probabilistic activation map for face recognition (see Supplemental Fig. S1) (Zhen et al., 2013), and the macroanatomical landmark reference was provided by the MNI152 T1 template. The first map provided information on subject-specific activations, whereas the latter two provided the functional and macro-anatomical landmarks for the location and extent of the FSRs. A semi-automated delineation procedure was followed by all raters, illustrated in Fig. 1. First, the subject-specific activation image for faces versus objects was thresholded with $Z=2.3$ ( $p=0.01$, right tailed, uncorrected) and then overlaid on the FSR spatial reference map (Fig. 1A). The thresholded $\mathrm{Z}=2.3$ was adopted in light of empirical evidence suggesting that a lenient statistical threshold could help to identify functional regions in cases of relatively small amounts of data (Kawabata Duncan and Devlin, 2011). Second, the thresholded activation image was partitioned into many small parcels using the watershed algorithm (Meyer, 1994) (Fig. 1B). Finally, the rater handpicked the small parcels from the watershed to construct the target regions based simultaneously on the FSR spatial reference and the macroanatomical landmark reference (Figs. 1B and C). A specialized tool, called FreeROI, was developed in Python to aid the raters in delineating regions of interest (ROI) with our procedure. The tool is freely available from the website of Brain Activity Atlas (http://www.brainactivityatlas. org).

Our semi-automated delineation procedure has three major advantages over the traditional handpicking method. First, the boundaries between parcels are objectively determined with the watershed algorithm, which avoids subjectivity in determining the boundaries of the FSRs. Second, guided by the spatial reference of the FSRs and macroanatomical landmark references, the FSRs could be identified more accurately. Third, delineating the FSRs in parcel units rather than in voxel units as in the traditional method can save a great deal of time.

To speed up the delineation and avoid biases from individual raters, seven raters took part in delineating the FSRs in four rounds. In the first round, the brains were randomly divided into seven approximately equal groups and each rater was assigned to delineate the FSRs for a group of brains. In the second round, each rater was assigned to delineate the FSRs for another group of brains. That is, following this round, each FSR had been delineated twice with two different raters. In the third round, the delineated FSRs from the first two rounds were double checked and refined by the two raters who had delineated them. Finally, the FSRs that failed to reach the agreement of the two raters were evaluated and then finalized by all seven raters together.

\section{Reliability of intra- and inter-rater delineations}

To evaluate the reliability of the delineation procedure, the interrater reliability was assessed based on the delineations from the first two rounds in which the same FSRs were delineated by two different raters. The inter-rater reliability of delineation was measured with Dice's coefficient, which is defined as the ratio of twice the number of overlapping voxels from two the delineations, divided by the total number of voxels present in both delineations. That is, given two different delineations of an FSR, denoted by $\mathrm{L}_{1}$ and $\mathrm{L}_{2}$, the Dice's coefficient is given by:

$\mathrm{O}\left(L_{1}, L_{2}\right)=\frac{2 * V\left(L_{1} \cap L_{2}\right)}{V\left(L_{1}\right)+V\left(L_{2}\right)}$ where $V(L)$ indicates the volume of L. For two identical delineations, Dice's coefficient achieves its maximum value of 1 , with decreasing values indicating less perfect overlap and 0 corresponding to no overlap.

To provide a baseline for the size of inter-rater variability, the intrarater reliability of delineation was also assessed. For this, each rater redelineated several weeks later the FSRs that she/he had finished in the first run. The re-delineated FSRs and the FSRs from the first run were used to compute the intra-rater reliability of delineation with Dice's coefficient as described above. A paired $t$-test was used to test the difference between the inter-rater and intra-rater reliability of delineation.

\section{Characterization of interindividual variability}

We next created a probabilistic atlas to characterize the locations and extents (or borders) of the FSRs precisely, and then quantified the variability of the FSRs on both functional (i.e., face selectivity) and spatial (i.e., volume, location of peak activation, and functional anatomy) features.

\section{Probabilistic atlas}

For each FSR, a probabilistic map was created to characterize the likelihood that a given voxel belonged to that FSR. Specifically, the subject-specific FSR delineated in individual brains were averaged in the MNI152 space so that the value at any voxel coded the likelihood of the voxel being located in the FSR, thus giving a measure of the variability in location and extent of the FSR over subjects at voxellevel resolution.

Because of the interindividual variability, the probabilistic maps from adjacent FSRs often showed some overlap. A maximumprobability map (MPM) was constructed to summarize the probabilistic maps of all FSRs into one volume. Specifically, the MPM was constructed by comparing the probabilities for each FSR (i.e., the overlapping frequency) in each voxel and assigning that voxel to the FSR to which it had the highest probability of belonging. If two FSRs showed equal probabilities, the problematic voxel was assigned to the FSR with the higher average probability in the 26 immediately adjacent voxels. The voxels with a maximal probability smaller than $10 \%$ were set to 0 , indicating that they most likely did not belong to any FSR. As a result, the MPM defined the most likely FSR to which each voxel belonged and represented all FSRs in a continuous, but non-overlapping manner in one volume.

\section{Face selectivity}

Face selectivity was characterized as percent signal change (PSC) for the contrast of faces versus objects. Specifically, the PSC was first calculated for each voxel by dividing the estimates of the parameters (the contrast of beta values from the fixed-effects GLM) by the mean intensity of the baseline level (the intercept in the GLM), and multiplying by 100. The PSC for an FSR was then calculated as the mean of PSC values across all voxels within the FSR. The interhemispheric difference in face selectivity was assessed with the paired $t$-test, and the effect size was measured with Cohen's d. Moreover, interindividual variability in face selectivity was quantified by the coefficient of variation (CV $=$ $\mathrm{SD} /$ mean) separately for each FSR. Permutation tests were used to test the significance of hemispheric effects on the CV of the magnitude.

\section{Volume}

The volume of an FSR was measured as the number of voxels within the FSR multiplied by the voxel size within the MNI space. The region size measured in the MNI space is equivalent to normalizing individual region volumes by total intracranial volume (TIV) and therefore avoids the confounding effect of TIV variation (Buckner et al., 2004). For each FSR, a significant effect of hemisphere on the volume was tested using the paired $t$-test, and the effect size was measured with Cohen's $\mathrm{d}$. Moreover, interindividual variability in volume was quantified by the 
CV separately for each FSR. Permutation tests were used to test the significance of hemispheric effects on the CV of the volume.

\section{Location of the peak activation}

The spatial locations of the FSRs were first characterized based on the MNI stereotaxic coordinates of the peak activation of each FSR. For each FSR, the coordinates of the peak activation were identified in each subject, and the mean coordinates of all peaks were calculated as the center of the peak locations across subjects. For each coordinate axis, differences of the absolute peak coordinates were tested for significant effects of hemisphere using the paired $t$-test. The dispersion of FSR peak locations on a given axis was measured as standard deviation (SD). Moreover, the sum of the three SDs was calculated to summarize the overall variability of peak location in MNI stereotaxic space. Permutation tests were used for each FSR to test for significant hemispheric effects on the total variation in the peak location of the FSRs.

\section{Anatomical labeling}

To supplement the stereotaxic coordinates of peak activation, the anatomical locations of the FSRs were also labeled in terms of the macro-anatomical regions defined in the Harvard-Oxford cortical probabilistic atlases (Desikan et al., 2006). First, the macro-anatomical regions were derived from the MPM of the Harvard-Oxford cortical probabilistic atlases implemented in FSL, without thresholding. Second, for each subject, the spatial overlap between each FSR and each anatomical region was indexed as a percentage of the FSR volume. Third, the spatial overlap index was averaged across subjects to characterize the correspondence between the FSRs and anatomical regions. The anatomical regions that had little overlap (less than 5\%) were considered non-stably overlapping and were discarded.

\section{Results}

\section{Delineation of FSRS}

The subject-specific FSRs were delineated based on the individual activation map with a parcel-based delineation procedure. As shown in Fig. 2A, the face-selective activation showed dramatic variation in the location, extent, and strength across individual brains. With our specialized tool, FreeROI, approximately 10 min were required for an expert to delineate all 12 FSRs in a brain. The OFA, pFFA, aFFA, pcSTS, pSTS, and aSTS in the right and left hemispheres were identified in 189/169, 198/187, 113/107, 169/140, 201/192, and 191/176 of 202 brains, respectively (Table 1 ). The delineated FSRs corresponding to the brain in Fig. 2A were shown in Fig. 2B, which clearly show the interindividual variability of the FSRs in size, location, and extent.

\section{Reliability of the delineation of FSRs}

The reliability of the delineation was measured using the Dice's coefficient. As shown in Fig. 3, all FSRs showed good intra-rater reliability with Dice's coefficients approximately equal to or exceeding 0.7. Particularly, OFA, pFFA, and pSTS showed intra-rater reliability close to or higher than 0.9 . The inter-rater reliability of the delineation is comparable to the intra-rater reliability, with Dice's coefficients exceeding 0.7 in most of the FSRs except left pcSTS, left aSTS, and right aSTS. No significant difference between inter- and intra-rater reliability was found ( $p<0.05$, Bonferroni correction). Remarkably, the reliability is comparable to the reported reliabilities of some manually labeled anatomical regions, such as the amygdala and hippocampus (Entis et al., 2012; Geuze et al., 2005), though the boundaries of the functional regions were not as clear as the boundaries of the anatomical regions. In summary, the raters had stable performance while delineating each FSR, and the delineations from different raters were highly consistent.

\section{Probabilistic atlas}

To characterize the interindividual variability of the locations and extents (or borders) of the FSRs, a probabilistic atlas of the FSRs was created by calculating the probability of a respective FSR being present at a given position. As a result, the probabilistic atlas consists of a set of 12 probability maps that reflect the interindividual variability of the respective FSRs. As shown in Fig. 4A, a high interindividual variability was observed for all FSRs: no one voxel showed a 100-percent chance of being in one of the FSRs across subjects. The maximum probability that a voxel belonged to an FSR ranged from 0.24 (left aFFA) to 0.74 (right pSTS) (Table 1). For each FSR, high probability was often found in the center, not the periphery. In addition, voxels in the center of the right FSRs usually had a larger probability than those of their left counterparts, especially for pFFA, pcSTS, and pSTS (Fig. 4A), suggesting
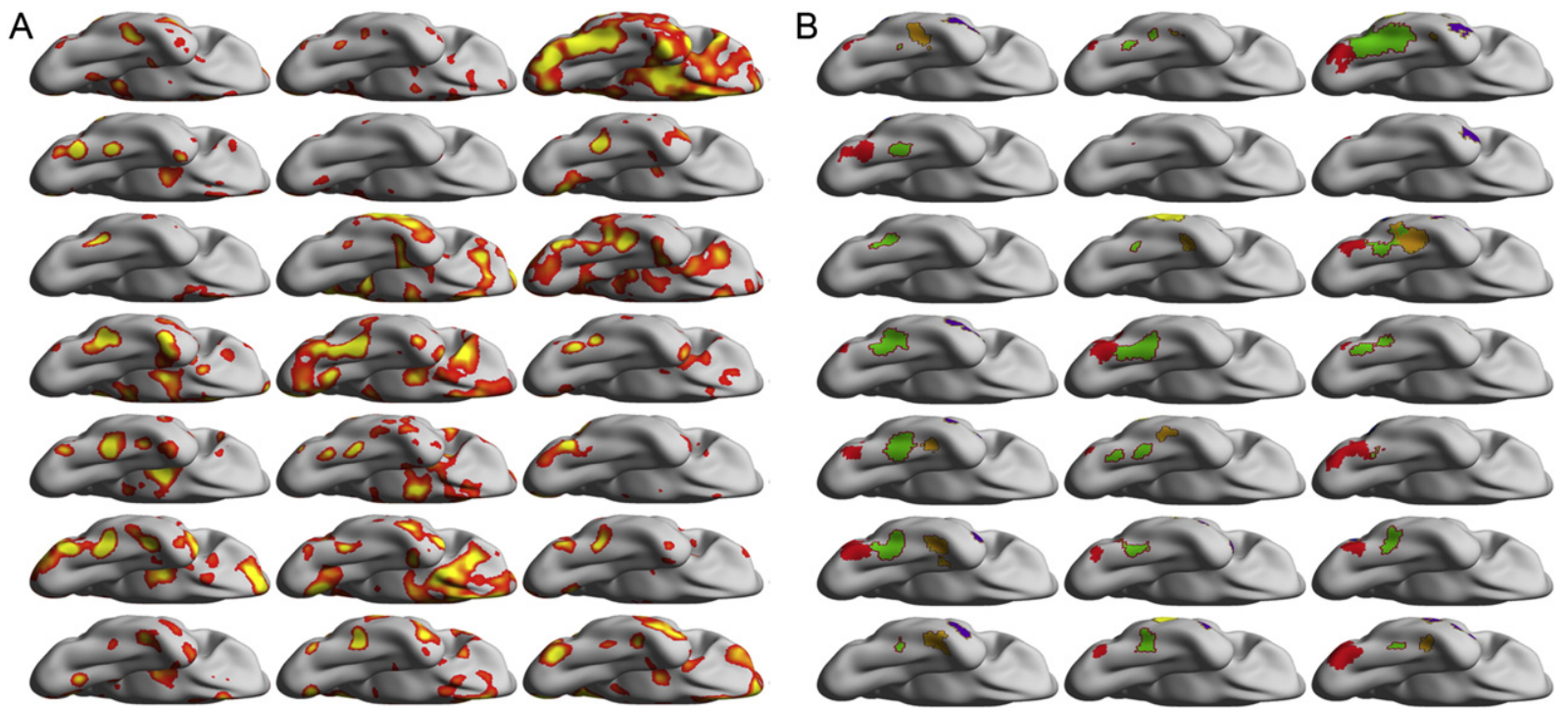

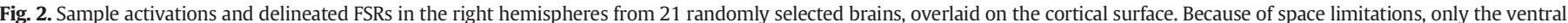

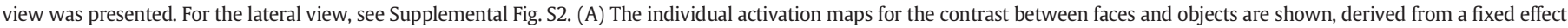

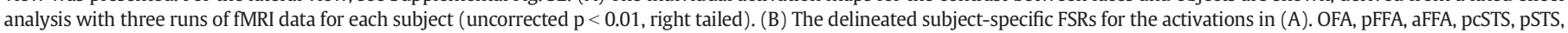
and aSTS were shown in red, green, ocher, blue, yellow, and purple, respectively. Each cell corresponds to one subject. 
Table 1

Characterization of face-selective regions: maximum probability, volume, coordinates, and selectivity.

\begin{tabular}{|c|c|c|c|c|c|c|c|}
\hline \multirow[t]{2}{*}{ Region name } & \multirow[t]{2}{*}{ Percent subjects } & \multirow[t]{2}{*}{ Maximum probability } & \multirow{2}{*}{$\begin{array}{l}\text { Volume }\left(\mathrm{cm}^{3}\right) \\
\text { Mean }(\mathrm{SD})\end{array}$} & \multicolumn{3}{|c|}{ MNI coordinates mean (SD) } & \multirow[t]{2}{*}{ Selectivity mean (SD) } \\
\hline & & & & $\bar{X}$ & $\mathrm{Y}$ & $\mathrm{Z}$ & \\
\hline R OFA & 93.56 & 0.52 & $4.86(4.29)$ & $43(6)$, & $-78(7)$ & $-13(5)$ & $0.54(0.15)$ \\
\hline L OFA & 83.66 & 0.32 & $3.15(3.85)$ & $-41(5)$ & $-80(6)$ & $-12(6)$ & $0.48(0.12)$ \\
\hline $\mathrm{R}$ pFFA & 98.02 & 0.64 & $3.70(3.32)$ & $42(4)$ & $-52(6)$ & $-20(5)$ & $0.55(0.14)$ \\
\hline L pFFA & 92.57 & 0.44 & $2.44(2.39)$ & $-40(3)$ & $-54(7)$ & $-20(5)$ & $0.46(0.12)$ \\
\hline R aFFA & 55.94 & 0.27 & $1.90(1.67)$ & $43(3)$ & $-24(7)$ & $-25(6)$ & $0.29(0.07)$ \\
\hline L aFFA & 52.97 & 0.24 & $1.22(1.11)$ & $-42(2)$ & $-26(7)$ & $-23(4)$ & $0.26(0.06)$ \\
\hline $\mathrm{R}$ pcSTS & 83.66 & 0.35 & $4.07(3.3)$ & $55(7)$ & $-59(5)$ & $7(7)$ & $0.55(0.14)$ \\
\hline L pcSTS & 69.31 & 0.27 & $2.42(2.33)$ & $-57(6)$ & $-62(5)$ & $9(6)$ & $0.40(0.11)$ \\
\hline R pSTS & 99.50 & 0.74 & $8.40(5.3)$ & $54(7)$ & $-38(6)$ & $4(6)$ & $0.53(0.11)$ \\
\hline L pSTS & 95.05 & 0.43 & $5.49(5.07)$ & $-58(8)$ & $-41(9)$ & $4(7)$ & $0.38(0.09)$ \\
\hline $\mathrm{R}$ aSTS & 94.55 & 0.47 & $4.85(4.03)$ & $55(6)$ & $-7(12)$ & $-15(8)$ & $0.38(0.08)$ \\
\hline L aSTS & 87.13 & 0.33 & $3.80(3.43)$ & $-58(6)$ & $-6(10)$ & $-16(8)$ & $0.33(0.08)$ \\
\hline
\end{tabular}

OFA, occipital face area; FFA, fusiform face area; STS, superior temporal sulcus; L, left; R, right; a, anterior; p, posterior; pc, posterior continuation.

that the FSRs in the left hemisphere have a larger variability in terms of anatomical location.

Because of the large interindividual variability, the probabilistic maps of adjacent FSRs showed overlaps to some extent in the periphery. To classify each voxel to the most likely region, an MPM was created to obtain a non-overlapping representation of the FSRs. As shown in Fig. 4B, the MPM represents multiple FSRs in one volume, which reveals a basic topographical pattern of these FSRs. On the lateral surface, the pcSTS was located in the posterior continuation of the STS, and abutted the OFA and the pSTS. The pSTS occupied the posterior STS, extending along STS to the aSTS. The aSTS occupied the anterior STS and extended along STS to the temporal pole. The OFA was located in the IOG and extended to the ventral surface. On the ventral surface, anterior to the OFA, the pFFA was located in the middle FG, and the aFFA was located more anteriorly in the FG. To avoid bias from a single arbitrary threshold, we also constructed the probabilistic atlas for the FSRs using other commonly used $\mathrm{Z}$ thresholds such as 3.09 (i.e., $\mathrm{p}=0.001$, right tailed, uncorrected) and 3.71 (i.e., $\mathrm{p}=0.0001$, right tailed, uncorrected) (see Supplemental Fig. S3). However, for simplicity only the FSRs delineated at $Z=2.3$ ( $p=0.01$, right tailed, uncorrected) were used for further analysis. Next, we further quantified the interindividual variability of the FSRs with respect to four features: face selectivity, volume, location of peak activation, and anatomical location.

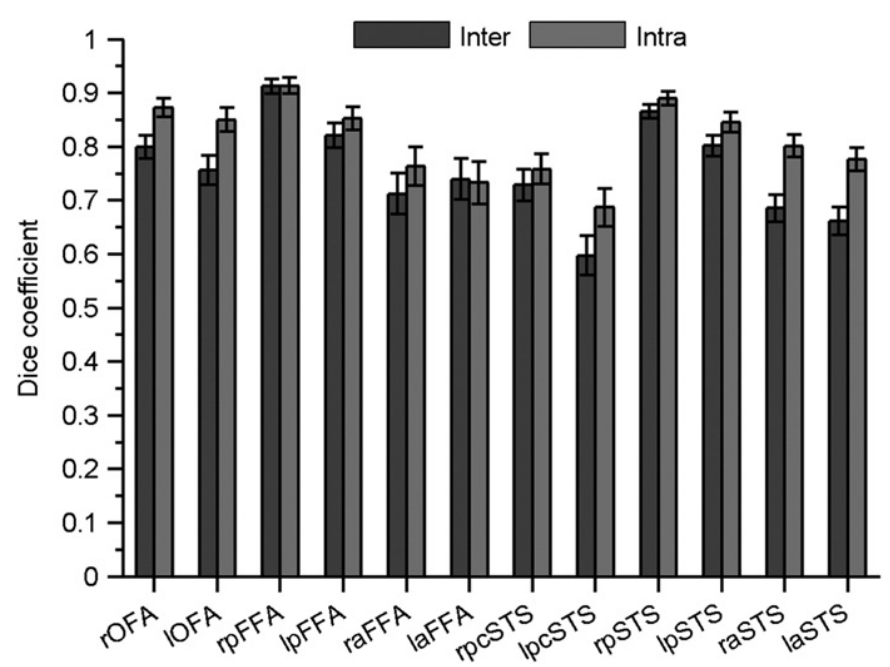

Fig. 3. Intra- and inter-rater reliabilities for the delineation of FSRs measured by the Dice's coefficient. Each FSR's reliability was calculated separately for its right and left component. Error bars indicate the standard deviation (SD) of the Dice's coefficients across subjects.

\section{Face selectivity}

The face selectivities of the FSRs were measured by percent signal change (PSC) from the contrast of faces versus objects, and its distribution was summarized in the box plots (Fig. 5A). Several interesting facts were observed. First, the magnitude of selectivity decreased gradually from posterior to anterior portions of both the lateral (pcSTS: $0.48 \pm$ 0.10 , pSTS: $0.45 \pm 0.08$, and aSTS: $0.35 \pm 0.07$ ) and ventral (OFA: $0.51 \pm 0.11$, pFFA: $0.51 \pm 0.11$, and aFFA: $0.27 \pm 0.06)$ temporal cortices. Second, the FSRs in the right hemisphere showed larger face selectivity than those in the left hemisphere (i.e., rightward asymmetry). The lateral FSRs (pSTS: $t(161)=17.40, p<0.001$, Cohen's $d=1.26$, pcSTS: $\mathrm{t}(183)=10.96, \mathrm{p}<0.001$, Cohen's $\mathrm{d}=0.98$, and aSTS: $\mathrm{t}(123)=7.98, \mathrm{p}<0.001$, Cohen's $\mathrm{d}=0.61)$ generally showed more rightward asymmetry than the ventral FSRs (OFA: $\mathrm{t}(170)=4.82$, $\mathrm{p}<0.001$, Cohen's $\mathrm{d}=0.38$, pFFA: $\mathrm{t}(73)=8.60, \mathrm{p}<0.001$, Cohen's $\mathrm{d}=0.63$, and aFFA: $\mathrm{t}(191)=4.85, \mathrm{p}<0.001$, Cohen's $\mathrm{d}=0.56$ ) (Fig. $5 \mathrm{~A}$ ). The large rightward asymmetries of the face selectivity suggest that the right hemisphere is dominant in face recognition.

Large interquartile ranges (IQR) were observed in the face selectivity for all FSRs, indicating that the FSRs had a significant amount of interindividual variability in their responses (Fig. 5A). The variability was further quantified by the CV. On average, across hemispheres, the FSRs showed CVs around 0.2 (OFA: 0.22, pFFA: 0.21, aFFA: 0.22, pcSTS: 0.20 , aSTS: 0.20 , and pSTS: 0.17 ) (Fig. 5B). A significant effect of hemisphere was observed in aSTS, with larger variation in the left than in the right hemisphere (CV difference $=-0.06, \mathrm{p}<0.001$ ). No significant interhemispheric differences in the variability were observed in other FSRs (all ps > 0.05).

Volume

The volume of each FSR was measured in the MNI space, and its distribution is summarized in the box plots in Fig. 6A. Averaging across the hemispheres, the pSTS had the largest volume (median: $6.89 \mathrm{~cm}^{3}$ ) while aFFA had the smallest volume (median: $1.33 \mathrm{~cm}^{3}$ ). The remaining FSRs had intermediate volumes (aSTS: $4.15 \mathrm{~cm}^{3}$, OFA: $3.77 \mathrm{~cm}^{3}$, pcSTS: $3.11 \mathrm{~cm}^{3}$, and pFFA: $3.01 \mathrm{~cm}^{3}$ ). Interhemispheric differences were observed in the FSRs: all right FSRs had larger volumes than their left counterparts did. Interestingly, the lateral FSRs (pSTS: $\mathrm{t}(191)=8.54$, $\mathrm{p}<0.001$, Cohen's d $=0.62$, pcSTS: $\mathrm{t}(123)=5.95, \mathrm{p}<0.001$, Cohen's $\mathrm{d}=0.53$, and aSTS: $\mathrm{t}(170)=3.64, \mathrm{p}<0.001$, Cohen's $\mathrm{d}=0.29$ ) generally showed larger interhemispheric differences in volume than the ventral FSRs (pFFA: $\mathrm{t}(183)=6.43, \mathrm{p}<0.001$, Cohen's $\mathrm{d}=0.47$, OFA: $\mathrm{t}(161)=5.76, \mathrm{p}<0.001$, Cohen's $\mathrm{d}=0.45$, and aFFA: $\mathrm{t}(73)=$ 3.89, $\mathrm{p}<0.001$, Cohen's $\mathrm{d}=0.45$ ) (Fig. 6A). 
A L
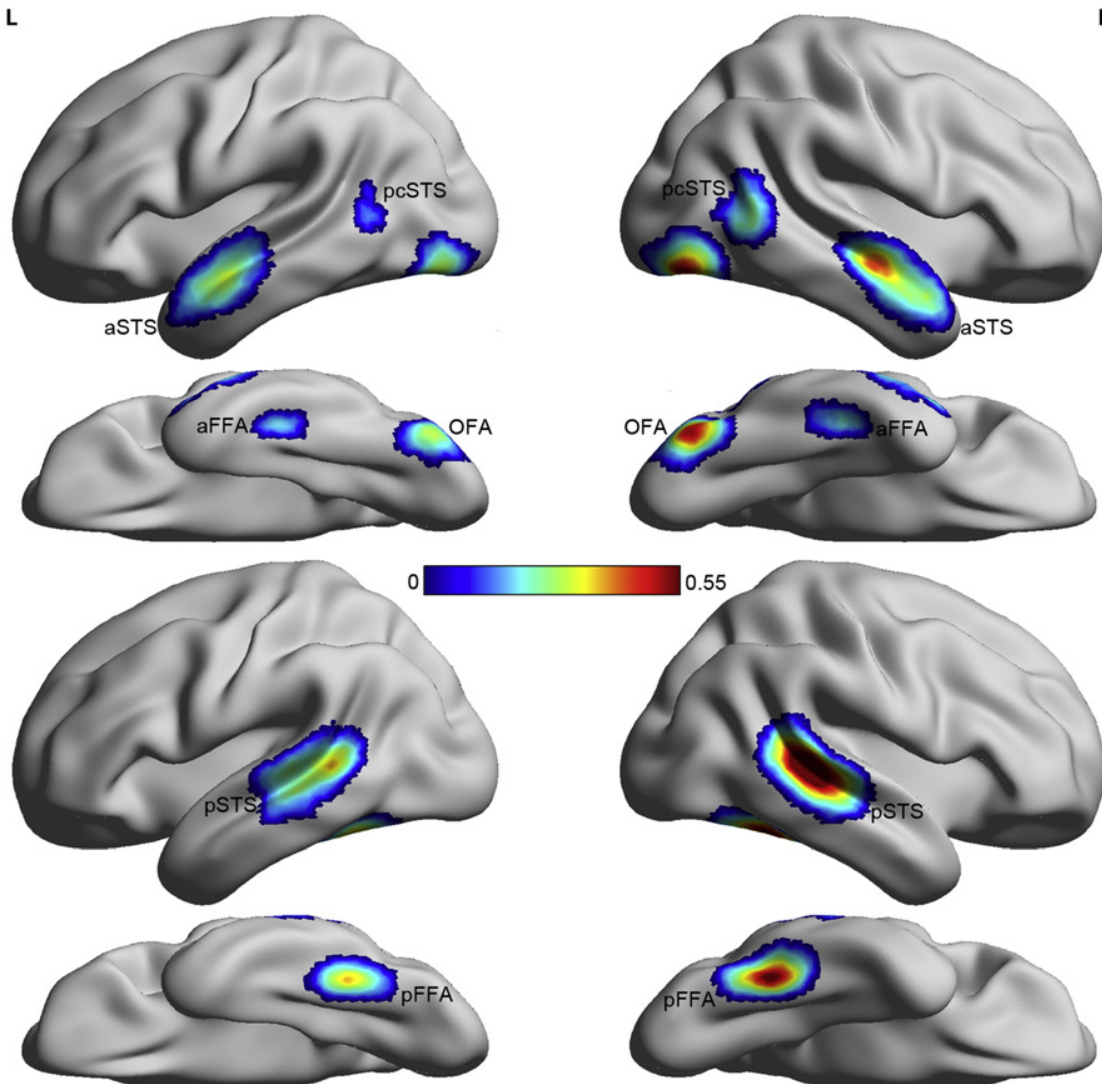

B
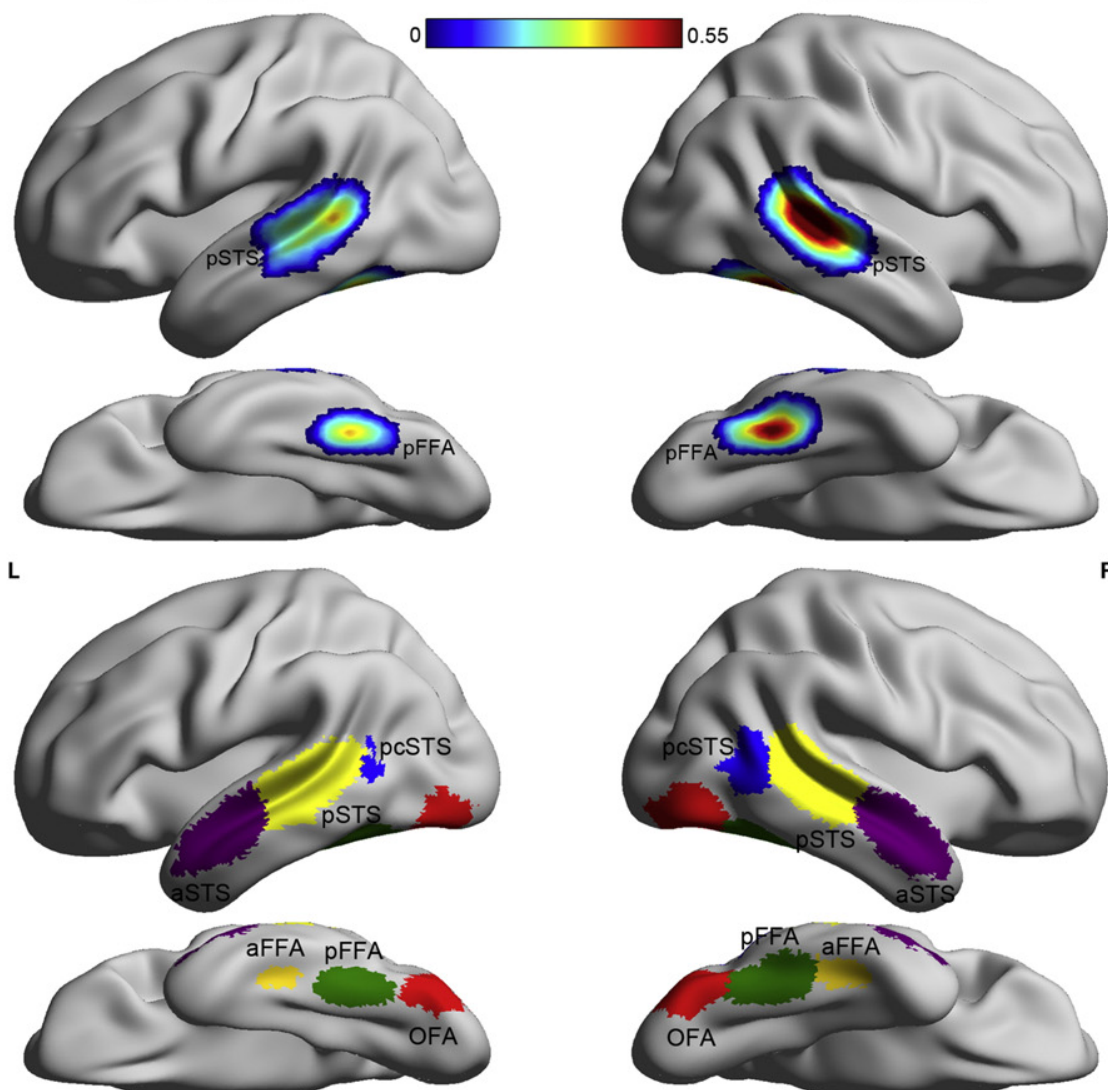

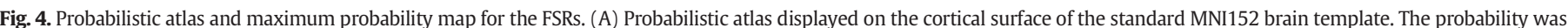

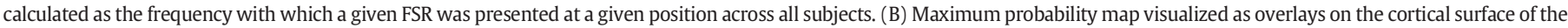

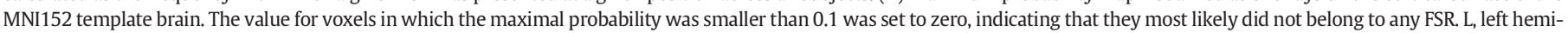
sphere; $\mathrm{R}$, right hemisphere.

Similar to face selectivity, large interquartile ranges (IQR) were observed in the distribution of the volumes for each FSR, indicating strong interindividual variation in the volume of all FSRs. The interindividual variability in the volume was further quantified by the CV. All FSRs showed large variations (OFA: 0.90, pFFA: 0.84, aFFA: 0.77, pcSTS: 0.74, pSTS: 0.67, and aSTS: 0.75). Moreover, as shown in Fig. 6B, all FSRs showed somewhat larger variations in volume in the left than in the right hemisphere. A significant leftward asymmetry was observed in OFA (CV diff $=-0.34, \mathrm{p}<0.001)$ and $\mathrm{pSTS}(\mathrm{CV}$ diff $=-0.29$, $\mathrm{p}<0.001)$.

Location of activation peak

To characterize the location of an FSR and its variability, the mean and SD of the stereotaxic coordinates of the peak activation were calculated in each axis (Table 1). As shown in Fig. 7A, despite considerable interindividual variations, the locations of the activation peaks of all FSRs differed significantly from each other along all three axes. Significant interhemispheric differences in peak location were observed in the X-coordinate in all FSRs, but with different sign. The OFA $(\mathrm{t}(162)=3.41, \mathrm{p}<0.001$, Cohen's $\mathrm{d}=0.27)$, pFFA $(\mathrm{t}(184)=5.16$, $\mathrm{p}<0.001$, Cohen's $\mathrm{d}=0.38)$, and aFFA $(\mathrm{t}(74)=2.46, \mathrm{p}=0.016$, Cohen's $d=0.29$ ) showed significantly larger absolute X-coordinates in the right hemisphere than in the left, indicating that they are more lateral in the right hemisphere than in the left hemisphere. In contrast, the pcSTS $(\mathrm{t}(124)=2.38, \mathrm{p}=0.019$, Cohen's $\mathrm{d}=-0.21)$, pSTS $(\mathrm{t}(192)=5.72, \mathrm{p}<0.001$, Cohen's $\mathrm{d}=-0.41)$, and aSTS $(\mathrm{t}(171)=$ $5.70, \mathrm{p}<0.001$, Cohen's $\mathrm{d}=-0.44)$ showed the reverse pattern (Fig. 7B), indicating that they are more lateral in the left hemisphere than in the right hemisphere. Significance in coordinate difference 

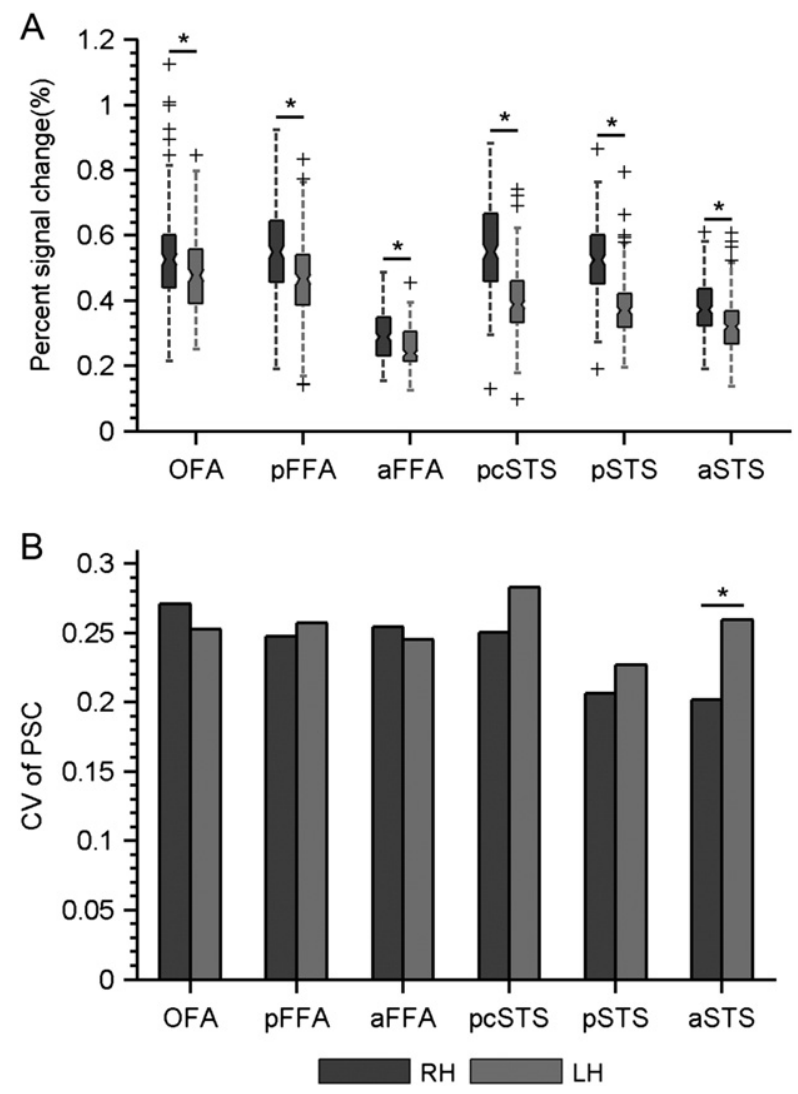

Fig. 5. The distribution, asymmetry, and variability of face selectivity. (A). The distribution of face selectivity revealed considerable interindividual and interhemispheric differences. The significance of the interhemispheric asymmetry was indicated by the horizontal lines on top of the boxplots. (B) Interindividual variation in face selectivity, quantified by the CV. Asterisks indicate $\mathrm{p}<0.05$.

was also found in the $\mathrm{Y}$ - and $\mathrm{Z}$-coordinates. In the Y-coordinate, the right OFA $(\mathrm{t}(162)=4.21, \mathrm{p}<0.001$, Cohen's $\mathrm{d}=0.33)$, pFFA $(\mathrm{t}$ $(184)=2.40, \mathrm{p}=0.018$, Cohen's $\mathrm{d}=0.18), \operatorname{pcSTS}(\mathrm{t}(124)=4.51$, $\mathrm{p}<0.001$, Cohen's $\mathrm{d}=0.40)$, and pSTS $(\mathrm{t}(192)=3.58, \mathrm{p}<0.001$, Cohen's $d=0.26$ ) were located more anteriorly than their left counterparts. In the Z-coordinate, the peaks of the right aFFA $(t(74)=2.77$, $\mathrm{p}=0.007$, Cohen's $\mathrm{d}=-0.32)$ and pcSTS $(\mathrm{t}(124)=2.61, \mathrm{p}=0.01$, Cohen's $d=-0.23$ ) were found more inferior than on the left (Fig. 7B).

The coordinates of the peak activation showed SDs from several millimeters to $1 \mathrm{~cm}$ in each axis and in each FSR (Fig. 7A). The sum of the SDs from three axes was calculated to quantify the overall variability of peak locations in stereotaxic space. As shown in Fig. 7C, the lateral FSRs (pcSTS: $14.0 \mathrm{~mm}$, pSTS: $16.3 \mathrm{~mm}$, and aSTS: $18.6 \mathrm{~mm}$ ) showed larger variations in peak coordinates than the ventral FSRs (OFA: $13.4 \mathrm{~mm}$, pFFA: $12.0 \mathrm{~mm}$, and aFFA: $11.7 \mathrm{~mm}$ ). A significant interhemispheric difference in variation was found only in pSTS, with larger variation in the left than in the right hemisphere (SD diff $=-3.85 \mathrm{~mm}$, $\mathrm{p}=0.001$ ).

\section{Anatomical correspondence}

Besides the coordinates of the FSRs, the anatomical locations of FSRs were also characterized in relation to the macro-anatomical regions of the Harvard-Oxford cortical probabilistic atlases (MPM with no threshold). The spatial overlap between the FSRs and the anatomical regions was examined. As shown in Fig. 8, there was no one-to-one correspondence between the FSRs and the Harvard-Oxford anatomical regions. An FSR usually overlapped with two or more anatomical regions. For example, the pFFA largely overlapped with the temporal occipital fusiform cortex $(52.9 \pm 25.2 \%)$, but also with the
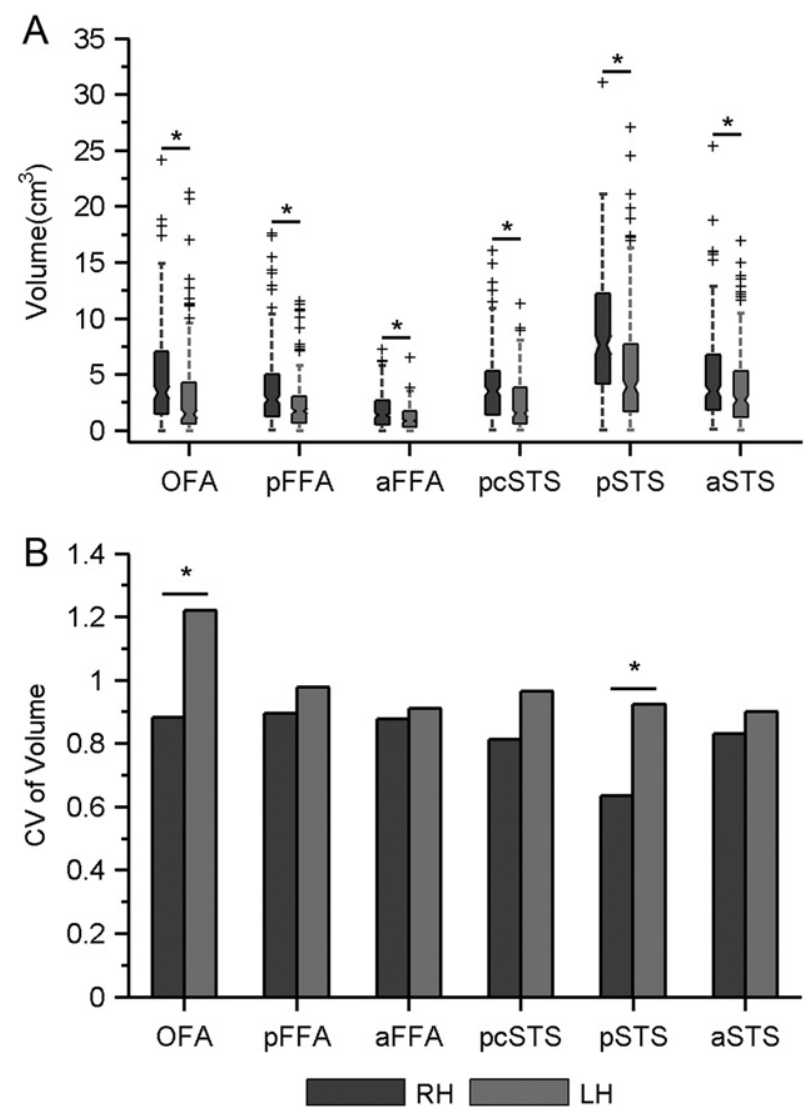

Fig. 6. The distribution, asymmetry, and variability for the volumes of the FSRs. (A) The distribution of the volumes revealed considerable interindividual and interhemispheric differences. The significance of the interhemispheric asymmetry was indicated by horizontal lines on top of the boxplots. (B) Interindividual variation for the volume of FSRs, quantified by the CV. Asterisks indicate $\mathrm{p}<0.05$.

temporooccipital part of the inferior temporal gyrus $(15.1 \pm 16.1 \%)$, the posterior division of the temporal fusiform cortex (7.38 $\pm 14.2 \%)$, and the occipital fusiform gyrus (7.22 $\pm 12.5 \%)$. Taken together, the results indicated that the locations of anatomical regions were not adequate to predict the locations of the FSRs.

\section{Discussion}

We have delineated the subject-specific FSRs in a large fMRI dataset and revealed that FSRs showed considerable variability across individuals and hemispheres with respect to face selectivity, volume, location of peak activation, and anatomical correspondence. Moreover, a probabilistic atlas was created to quantify the spatial variability of FSRs, which contained precise stereotaxic information on both interhemispheric and interindividual differences.

\section{Interindividual variability of the FSRs}

Our data revealed that the FSRs showed considerable interindividual variability in both functional and spatial features. As for the functional features, the FSRs showed significant variability in their selectivity for faces, consistent with previous studies (Furl et al., 2011; Gauthier et al., 2005; Yovel and Kanwisher, 2005). For the spatial features, to our knowledge, only a few studies have provided quantitative estimates of intersubject variability of the FSRs, mostly focusing on the variability of the location of peak activation. The interindividual variability of peak location of FSRs reportedly ranged from several millimeters to centimeters (Fox et al., 2009; Kanwisher et al., 1997; Pinsk et al., 2009). Moreover, the interindividual variability in the volume of FSRs 

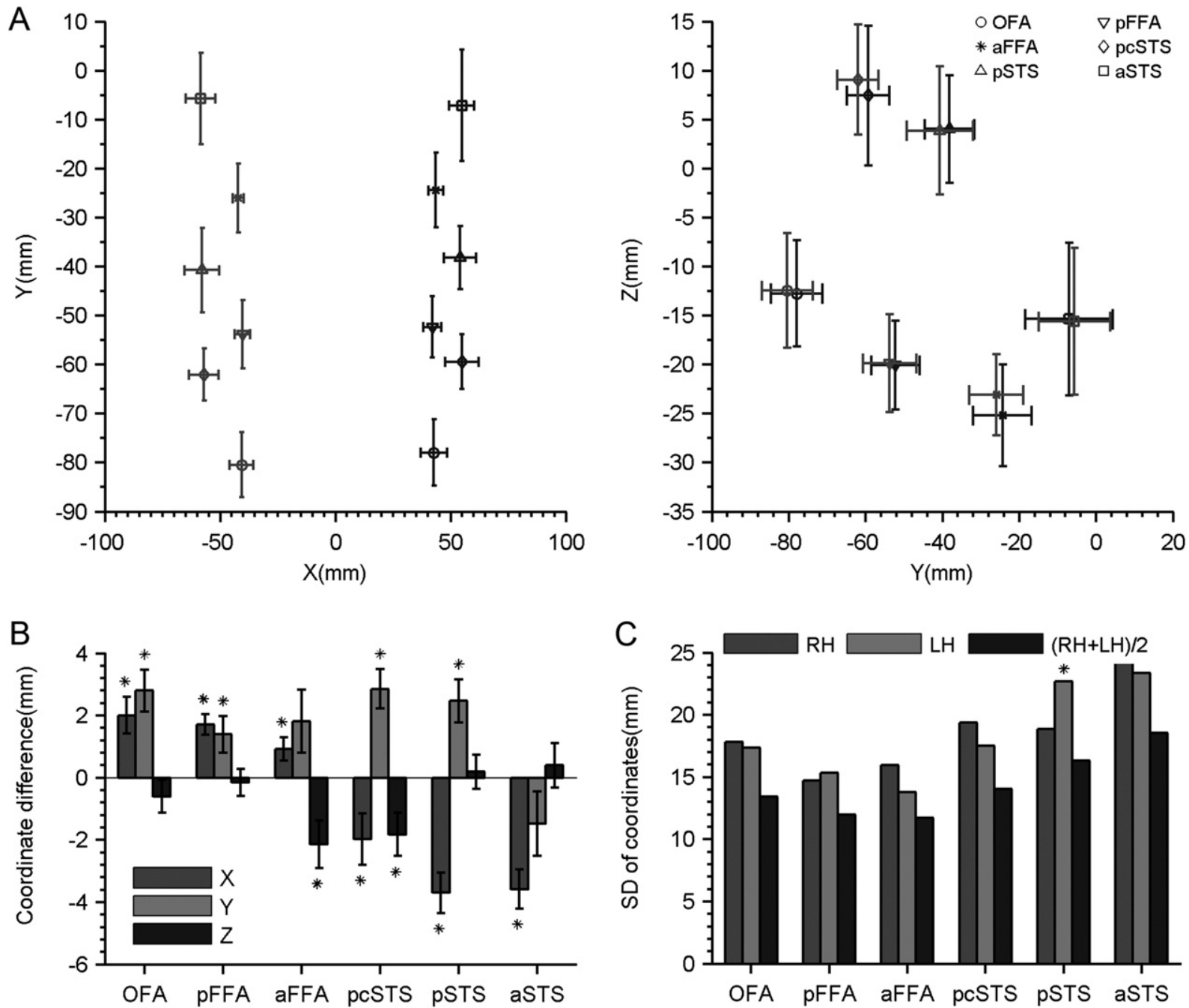

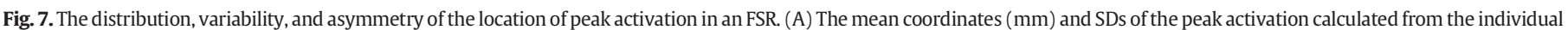

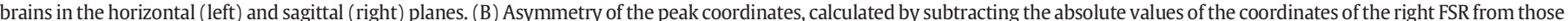
of the left. (C) Interindividual variations in the location of peak activation, quantified as the sum of the SDs of all three coordinate axes. Asterisks indicate $\mathrm{p}<0.05$.

was also examined either in subjects' native space (Kanwisher et al., 1997; Pinsk et al., 2009) or by fixing the size of the FFA at $200 \mathrm{~mm}^{3}$ (Rossion et al., 2012), resulting in a finding that the interindividual variability of the volume (i.e., the SD of the volume measurements) is approximately equal to or greater than the mean volume of each FSR. Our study extended these studies on the spatial features of the FSRs in three folds. First, the probabilistic atlas revealed that the FSRs had high uncertainty in location and extent: not one voxel in an FSR was found to show face selectivity in all participants tested. Second, the distance among the locations of peak activation across participants in an FSR was more than $10 \mathrm{~mm}$. Finally, interindividual variability in the volumes of the FSRs (CV: 0.67-0.90) was dramatically larger than that of the mean volume of the total neocortex (CV: 0.1) (Kennedy et al., 1998).

In our study, all individual brains were standardized to the MNI152 space with a high dimensional non-linear registration method, and thus the macro-anatomical variability across subjects was largely, if not wholly, removed. In fact, even with an arguably more accurate curvature-based alignment, the variability of functional regions remains prominent (Frost and Goebel, 2012). Therefore, the observed variability likely reflected the true functional variability (i.e., the structuralfunctional correspondence). Such interindividual functional variability likely provides a neural basis for interindividual differences in face recognition. For example, individual differences in face selectivity of the OFA and FFA predict differences in behavioral performance in face recognition (Furl et al., 2011; Huang et al., 2014). In addition, differences in the volume of the FFA were found to account for the developmental differences in face recognition memory (Golarai et al., 2007). The behavioral relevance of the interindividual variability of the FSRs located in the STS has not been extensively studied; we speculate that it might be related to individual differences in processing expression, eye gaze, or other dynamic aspects of faces.

\section{Sources of interindividual variability}

The interindividual functional variability may stem from multiple sources. First, the variability may simply relate to the microstructural organization (e.g., cytoarchitecture) of the cortex. In the human brain, it has been observed that the size and location of cytoarchitectonic visual areas varies considerably across individuals (Amunts et al., 2000; Caspers et al., 2013; Malikovic et al., 2007; Rottschy et al., 2007; Zilles and Amunts, 2013). Because the regional cytoarchitecture is often correlated with the regional specialization of function (Brett et al., 2002; Zilles and Amunts, 2010), the variability of the cytoarchitecture is thus likely a source of the variability in the functional regions. Second, as the function of a cortical region is largely determined by its extrinsic connections (Passingham et al., 2002; Saygin et al., 2011), the variability of its underlying anatomical and functional connectivity patterns may be another 


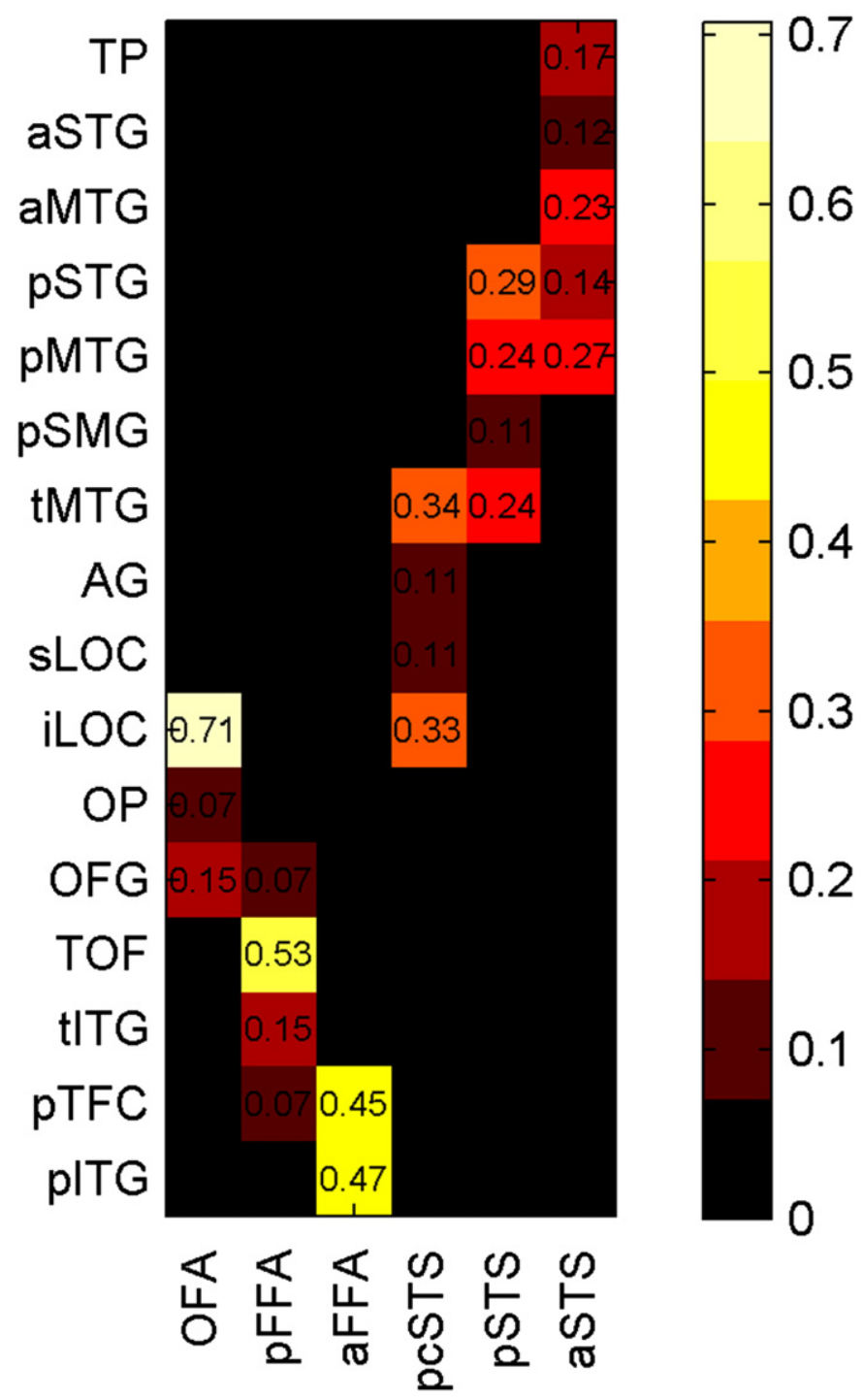

Fig. 8. The anatomical correspondence of the FSRs. The correspondence between functional and macro-anatomical regions was evaluated in terms of the spatial overlap between the FSRs and the MPM (no threshold) of the Harvard-Oxford cortical probabilistic atlas. The value indicated the percentage of the intersection volume to the whole volume of the FSRs (i.e., the number of overlapping voxels divided by the total number of voxels present in the FSR). For display purposes, the macro-anatomical regions that had little overlap (less than 10\%) were not shown. Abbreviations: TP, temporal pole; aSTG, superior temporal gyrus, anterior division; aMTG, middle temporal gyrus, anterior division; PSTG, superior temporal gyrus, posterior division; pSMG, supramarginal gyrus, posterior division; tMTG, middle temporal gyrus, temporooccipital part; AG, angular gyrus; SLOC, lateral occipital cortex, superior division; iLOC, lateral occipital cortex, inferior division; OP, occipital pole; OFG, occipital fusiform gyrus; TOF, temporal occipital fusiform cortex; tITG, inferior temporal gyrus, temporooccipital part; pTFC, temporal fusiform cortex, posterior division; pITG, inferior temporal gyrus, posterior division.

source of the variability in functional regions. Indeed, a recent study has revealed that the interregional functional connectivity shows considerable variability across subjects (Mueller et al., 2013). Third, the large interindividual functional variability may arise from the functional plasticity of the brain through cortical competition. Previous studies on visual development (Golarai et al., 2007), expertise (Gauthier et al., 2003), and plasticity in sensory maps (Polley et al., 2006; Song et al., 2010) have revealed a displacement of map boundaries due to cortical competition. Thus, individual experiences in the early or even late years of development may significantly contribute to the interindividual variability of the FSRs. Finally, a growing body of evidence indicates that individual variation in brain activation is significantly influenced by genetic factors (Blokland et al., 2011; Koten et al., 2009; Polk et al., 2007).
Therefore, the large variability of FSRs is likely to be a joint outcome of genetic and environmental factors. To what extent the variability of functional regions is determined by the two factors requires further investigation.

\section{Interhemispheric variability}

Considerable interhemispheric variability was observed in both functional and spatial features as well. Not surprisingly all right FSRs showed larger face selectivity and volumes than their left counterparts. Our results further revealed that the lateral FSRs generally showed more rightward asymmetry than the ventral FSRs. Moreover, as shown in the probabilistic atlas, the right FSRs usually showed less variability than their left counterparts did. In line with our observation, previous studies have reported the right-lateralization in face recognition. First, a bias for better retention and recognition of faces briefly presented to the left visual field is consistently observed in behavioral studies (Hillger and Koenig, 1991; Rhodes, 1985). Second, most, if not all, acquired prosopagnosics follow either bilateral or right unilateral occipitotemporal lesions (Bouvier and Engel, 2006; Landis et al., 1988). Third, neuroimaging (Kanwisher et al., 1997; McCarthy et al., 1997; Rossion et al., 2012) and ERP (Bentin et al., 1996; Rossion et al., 2003; Yovel et al., 2003) studies have also demonstrated the right hemispheric dominance for face recognition.

The reason of the right asymmetry in FSRs remains largely unknown (Bukowski et al., 2013). One possibility is the right hemispheric dominance in holistic processing (Bradshaw and Nettleton, 1981; Schiltz and Rossion, 2006). Because holistic processing is critical in identifying faces, the right FSRs may play a more important role in face processing. Another possibility is that the right asymmetry of FSRs may result from a competition between the processing of faces versus language (Knecht et al., 2000) in the left hemisphere. During development, the left FSRs might compete with nearby language-related regions, such as the visual word form area in the middle fusiform gyrus (Baker et al., 2007; Cohen et al., 2000; Song et al., 2012) and Wernicke's area near the STS (Calvert and Campbell, 2003). Consequently, the left FSRs would show reduced selectivity and volumes compared to their right counterparts that encountered less competition. However, neither possibility can satisfactorily explain the right asymmetry of the FSRs simply because not all FSRs are involved in holistic processing or have a neighboring language-related area (e.g., the pcSTS). Future studies are needed to explore how asymmetry is formed during development, and whether there are different mechanisms underlying the asymmetry of different FSRs.

\section{Probabilistic functional atlas (PFA)}

Atlases of the human brain play an important role in brain research. An atlas captures the spatiotemporal distribution of a multitude of physiological and anatomical metrics in a stereotaxic space (Evans et al., 2012; Toga et al., 2006). A series of brain atlases has been created and widely used (Evans et al., 2012; Mazziotta et al., 2001; Mori et al., 2008; Shattuck et al., 2008; Zilles and Amunts, 2010). However, most of the existing atlases focus on brain anatomy, not brain functions. Because of great intersubject variability in anatomy and structuralfunctional correspondence, the anatomical atlases are not sufficient to allow researchers to infer the location and extent of functional regions and their variability across individual brains (Frost and Goebel, 2012). The lack of brain atlases for functional regions renders it difficult to accumulate accurate knowledge of specific functional regions and to understand the structural and functional architecture of the face processing system.

One contribution of our study is the construction of a probabilistic functional atlas (PFA) for the FSRs based on a large cohort of subjects. The PFA could serve as a useful repository of knowledge and facilitate the analysis of fMRI data (Evans et al., 2012; Mazziotta et al., 2001). 
First, as a complement to the anatomical atlases, the PFA supplies a quantitative spatial reference system in which information from multiple sources can be integrated and compared. Moreover, by integrating with other atlases, the PFA would identify patterns of structural, connectional, and molecular variations in the functional regions and provide a deeper understanding of the relationship between brain structure and function. Second, subject-specific fROIs are always preferred over the PFA based fROIs in individual studies; however, when such information is not available, the PFA can be used to provide faceselective fROIs in group analyses to explore properties common to all human brains. On the other hand, even when a functional localizer is acquired, the PFA can be used as a source of spatial constraints to localize subject-specific fROIs. The availability of well-defined fROIs could provide better sensitivity and specificity in integrating features of the FSRs with clinical and genetic data, and in studying the large-scale brain network for face recognition. Third, the labeled individual FSRs and associated features could serve as a database of the FSRs in a healthy population. All the accumulated data in the atlas could be used as a norm to quantify the degree of deviation of the FSRs in a new subject and thereby have the potential to detect FSR deficits in patients. Fourth, the atlas descriptions of intersubject variance in FSRs could serve as empirical priors in the development of algorithms for automated identification of the FSRs. The automated algorithms will in turn improve the objectivity and efficiency of identification of the FSRs in new subjects.

Several groups have recently begun developing probabilistic atlases (or maps) to characterize face-selective activation (Engell and McCarthy, 2013; Julian et al., 2012; Tahmasebi et al., 2012). Our PFA significantly extended these atlases. First, previous atlases aim to describe the likelihood of activation of each voxel elicited by faces and thus provide little information on how the FSRs vary in location and extent across subjects (but see Frost and Goebel, 2012). Instead of characterizing the interindividual variability at the voxel level, our study summarized the subject-specific FSRs across subjects to classify face-selective voxels to a specific FSR. The atlas at the regional level likely provides information more directly related to cognitive processes of interest. Second, the delineation of multiple regions together provides information about the spatial and functional organizations of these regions, which makes comparison of their anatomical and functional relations possible. More broadly, the former type of atlases characterize the interindividual variability of activation for the cognitive process of interest at the voxel level, and the latter type of atlases characterize the interindividual variability of the functional regions that are involved in the cognitive process of interest. Therefore, the two types of atlases are not substitutes but complement each other. The two types of atlases together could be the cornerstone of functional atlases, in which brain regions are annotated according to their cognitive and behavioral involvements.

\section{Unwanted sources of variability}

It should be noted that some of the observed spatial variability might stem from unwanted sources of variability. First, although a high-dimensional non-linear registration method was used to remove macro-anatomical variability, some may remain. To more accurately measure the interindividual variability of functional regions, a more advanced alignment scheme needs to be developed to better remove macro-anatomical variability (Avants et al., 2011; Frost and Goebel, 2012, 2013), although perfect alignment of any two brains may not possible (Juch et al., 2005; Van Essen and Dierker, 2007). Second, the low power of the localizer data may cause some variability. Our functional localizer consists of three runs that take a relatively short scanning period ( $\sim 10 \mathrm{~min})$. Although results from previous studies have revealed that this amount of data can well localize the FSRs in individuals (70-80\% overlap with the gold standard from a randomeffects analysis of ten runs), the low signal-to-noise ratio of fMRI may still lead to some unreliable FSR identifications (Kawabata Duncan and
Devlin, 2011; Swallow et al., 2003). Third, the choice of the threshold in localizing the FSRs inevitably influenced the amount of variation in the location of the FSRs. To avoid bias from a single arbitrary threshold, we also constructed the PFA using other commonly used $\mathrm{Z}$ thresholds such as 3.09 (i.e., p $=0.001$, right tailed, uncorrected) and 3.71 (i.e., $p=0.0001$, right tailed, uncorrected) (see Supplemental Fig. S3), As shown, the probabilistic atlas and maximum probability map derived at different thresholds revealed similar patterns, indicating that our atlas is stable and not sensitive to the choice of threshold. Fourth, the inter-rater inconsistency in labeling is another factor potentially contributing unwanted variability, although our procedure of requiring the collaboration of two raters on each labeling dramatically reduced this type of variability. Moreover, as our reliability analysis noted, the reliability of our delineation had reached a good level, comparable to the reliabilities of some manually delineated anatomical regions, such as the amygdala and hippocampus (Entis et al., 2012; Geuze et al., 2005).

\section{Future directions}

The large variability of FSRs presented here suggests several avenues for future study. First, behavioral studies have revealed that the ability to recognize faces continues to develop until 33 years of age (Susilo et al., 2013), but the developmental trajectory of the FSRs is poorly known. The atlas presented in this study is constructed from young adults with a narrow range of ages; therefore, the atlas may not be applicable to studies on people with different ages, especially child participants. Future studies are needed to construct a functional atlas that reflects the development of functional cortical regions of the brain. Second, our definition of the FSRs relied on the functional contrast between faces and objects. When more information is introduced to define the FSRs, we may have more power to distinguish the different regions (e.g., pFus and mFus). For example, considering the connectivity patterns of regions of interest is a noteworthy future direction in refining the PFA for faces processing. Finally, the interindividual variability of functional regions in both functional and spatial features should reflect brain function to some extent, and thus is a rich and important source of information for revealing the neural basis of human cognition and behavior (Kanai and Rees, 2011; Zilles and Amunts, 2013). Future studies need to elaborate on the significance of the variability of functional regions and utilize this variability to explore the neural mechanisms of specific behavioral or cognitive processes (Van Horn et al., 2008).

\section{Conclusion}

Our study demonstrates large interindividual variability in the FSRs. This work is the first step toward identifying morphological landmarks potentially useful for correlating functional and structural maps, and for understanding the neural mechanism of individual differences in face recognition. Moreover, the probabilistic atlas of FSRs from this work is freely available (http://www.brainactivityatlas.org). It not only describes the commonalities in the population data, but also captures the interindividual variability in the location and extent of the FSRs. Thus, the atlas stands as a resource for the neuroimaging community and a stimulus for the future construction of probabilistic atlases of other functional regions and domains.

Supplementary data to this article can be found online at http://dx. doi.org/10.1016/j.neuroimage.2015.03.010.

\section{Acknowledgments}

This study was supported by the National Natural Science Foundation of China (31470055, 31230031, 91132703, and 31221003), the National Basic Research Program of China (2014CB846103) and the Fundamental Research Funds for the Central Universities. 


\section{Conflict of interest}

The authors declare no interests of conflict.

\section{References}

Allison, T., Puce, A., McCarthy, G., 2000. Social perception from visual cues: role of the STS region. Trends Cogn. Sci. 4, 267-278.

Amunts, K., Schleicher, A., Burgel, U., Mohlberg, H., Uylings, H.B., Zilles, K., 1999. Broca's region revisited: cytoarchitecture and intersubject variability. J. Comp. Neurol. 412, 319-341.

Amunts, K., Malikovic, A., Mohlberg, H., Schormann, T., Zilles, K., 2000. Brodmann's areas 17 and 18 brought into stereotaxic space-where and how variable? Neuroimage 11, $66-84$.

Avants, B.B., Tustison, N.J., Song, G., Cook, P.A., Klein, A., Gee, J.C., 2011. A reproducible evaluation of ANTs similarity metric performance in brain image registration. Neuroimage 54, 2033-2044.

Baker, C.I., Liu, J., Wald, L.L., Kwong, K.K., Benner, T., Kanwisher, N., 2007. Visual word processing and experiential origins of functional selectivity in human extrastriate cortex. Proc. Natl. Acad. Sci. U. S. A. 104, 9087-9092.

Bartley, A.J., Jones, D.W., Weinberger, D.R., 1997. Genetic variability of human brain size and cortical gyral patterns. Brain 120, 257-269.

Bentin, S., Allison, T., Puce, A., Perez, E., McCarthy, G., 1996. Electrophysiological studies of face perception in humans. J. Cogn. Neurosci. 8, 551-565.

Blokland, G.A., McMahon, K.L., Thompson, P.M., Martin, N.G., de Zubicaray, G.I., Wright, M.J., 2011. Heritability of working memory brain activation. J. Neurosci. 31, 10882-10890.

Bouvier, S.E., Engel, S.A., 2006. Behavioral deficits and cortical damage loci in cerebral achromatopsia. Cereb. Cortex 16, 183-191.

Bradshaw, J.L., Nettleton, N.C., 1981. The nature of hemispheric-specialization in man. Behav. Brain Sci. 4, 51-63.

Brett, M., Johnsrude, I.S., Owen, A.M., 2002. The problem of functional localization in the human brain. Nat. Rev. Neurosci. 3, 243-249.

Buckner, R.L., Head, D., Parker, J., Fotenos, A.F., Marcus, D., Morris, J.C., Snyder, A.Z., 2004. A unified approach for morphometric and functional data analysis in young, old, and demented adults using automated atlas-based head size normalization: reliability and validation against manual measurement of total intracranial volume. Neuroimage 23, 724-738.

Bukowski, H., Dricot, L., Hanseeuw, B., Rossion, B., 2013. Cerebral lateralization of facesensitive areas in left-handers: only the FFA does not get it right. Cortex 49, 2583-2589.

Burgel, U., Amunts, K., Hoemke, L., Mohlberg, H., Gilsbach, J.M., Zilles, K., 2006. White matter fiber tracts of the human brain: three-dimensional mapping at microscopic resolution, topography and intersubject variability. Neuroimage 29, 1092-1105.

Calvert, G.A., Campbell, R., 2003. Reading speech from still and moving faces: the neural substrates of visible speech. J. Cogn. Neurosci. 15, 57-70.

Caspers, S., Geyer, S., Schleicher, A., Mohlberg, H., Amunts, K., Zilles, K., 2006. The human inferior parietal cortex: cytoarchitectonic parcellation and interindividual variability. Neuroimage 33, 430-448.

Caspers, J., Zilles, K., Eickhoff, S.B., Schleicher, A., Mohlberg, H., Amunts, K., 2013. Cytoarchitectonical analysis and probabilistic mapping of two extrastriate areas of the human posterior fusiform gyrus. Brain Struct. Funct. 218, 511-526.

Cohen, L., Dehaene, S., Naccache, L., Lehericy, S., Dehaene-Lambertz, G., Henaff, M.A., Michel, F., 2000. The visual word form area: spatial and temporal characterization of an initial stage of reading in normal subjects and posterior split-brain patients. Brain 123, 291-307.

Desikan, R.S., Segonne, F., Fischl, B., Quinn, B.T., Dickerson, B.C., Blacker, D., Buckner, R.L., Dale, A.M., Maguire, R.P., Hyman, B.T., Albert, M.S., Killiany, R.J., 2006. An automated labeling system for subdividing the human cerebral cortex on MRI scans into gyral based regions of interest. Neuroimage 31, 968-980.

Engell, A.D., McCarthy, G., 2013. Probabilistic atlases for face and biological motion perception: an analysis of their reliability and overlap. Neuroimage 74, 140-151.

Entis, J.J., Doerga, P., Barrett, L.F., Dickerson, B.C., 2012. A reliable protocol for the manual segmentation of the human amygdala and its subregions using ultra-high resolution MRI. Neuroimage 60, 1226-1235.

Evans, A.C., Janke, A.L., Collins, D.L., Baillet, S., 2012. Brain templates and atlases. Neuroimage 62, 911-922.

Fischl, B., Dale, A.M., 2000. Measuring the thickness of the human cerebral cortex from magnetic resonance images. Proc. Natl. Acad. Sci. U. S. A. 97, 11050-11055.

Fischl, B., Rajendran, N., Busa, E., Augustinack, J., Hinds, O., Yeo, B.T., Mohlberg, H., Amunts, K., Zilles, K., 2008. Cortical folding patterns and predicting cytoarchitecture. Cereb. Cortex 18, 1973-1980.

Fox, C.J., Iaria, G., Barton, J.J.S., 2009. Defining the face processing network: optimization of the functional localizer in fMRI. Hum. Brain Mapp. 30, 1637-1651.

Frost, M.A., Goebel, R., 2012. Measuring structural-functional correspondence: spatial variability of specialised brain regions after macro-anatomical alignment. Neuroimage 59, 1369-1381.

Frost, M.A., Goebel, R., 2013. Functionally informed cortex based alignment: an integrated approach for whole-cortex macro-anatomical and ROI-based functional alignment. Neuroimage 83, 1002-1010.

Furl, N., Garrido, L., Dolan, R.J., Driver, J., Duchaine, B., 2011. Fusiform gyrus face selectivity relates to individual differences in facial recognition ability. J. Cogn. Neurosci. 23, $1723-1740$.
Gauthier, I., Tarr, M.J., Anderson, A.W., Skudlarski, P., Gore, J.C., 1999. Activation of the middle fusiform 'face area' increases with expertise in recognizing novel objects. Nat. Neurosci. 2, 568-573.

Gauthier, I., Tarr, M.J., Moylan, J., Skudlarski, P., Gore, J.C., Anderson, A.W., 2000. The fusiform "face area" is part of a network that processes faces at the individual level. J. Cogn. Neurosci. 12, 495-504.

Gauthier, I., Curran, T., Curby, K.M., Collins, D., 2003. Perceptual interference supports a non-modular account of face processing. Nat. Neurosci. 6, 428-432.

Gauthier, I., Curby, K.M., Skudlarski, P., Epstein, R.A., 2005. Individual differences in FFA activity suggest independent processing at different spatial scales. Cogn. Affect. Behav. Neurosci. 5, 222-234.

Geuze, E., Vermetten, E., Bremner, J.D., 2005. MR-based in vivo hippocampal volumetrics: 1. Review of methodologies currently employed. Mol. Psychiatry 10, 147-159.

Goffaux, V., Peters, J., Haubrechts, J., Schiltz, C., Jansma, B., Goebel, R., 2011. From coarse to fine? Spatial and temporal dynamics of cortical face processing. Cereb. Cortex 21, 467-476.

Golarai, G., Ghahremani, D.G., Whitfield-Gabrieli, S., Reiss, A., Eberhardt, J.L., Gabrieli, J.D. Grill-Spector, K., 2007. Differential development of high-level visual cortex correlates with category-specific recognition memory. Nat. Neurosci. 10, 512-522.

Hasnain, M.K., Fox, P.T., Woldorff, M.G., 1998. Intersubject variability of functional areas in the human visual cortex. Hum. Brain Mapp. 6, 301-315.

Haxby, J.V., Hoffman, E.A., Gobbini, M.I., 2000. The distributed human neural system for face perception. Trends Cogn. Sci. 4, 223-233.

Hillger, L.A., Koenig, O., 1991. Separable mechanisms in face processing: evidence from hemispheric specialization. J. Cogn. Neurosci. 3, 42-58.

Hoffman, E.A., Haxby, J.V., 2000. Distinct representations of eye gaze and identity in the distributed human neural system for face perception. Nat. Neurosci. 3, 80-84.

Huang, L., Song, Y., Li, J., Zhen, Z., Yang, Z., Liu, J., 2014. Individual differences in cortical face selectivity predict behavioral performance in face recognition. Front. Hum. Neurosci. 8.

Ishai, A., 2008. Let's face it: it's a cortical network. Neuroimage 40, 415-419.

Juch, H., Zimine, I., Seghier, M.L., Lazeyras, F., Fasel, J.H., 2005. Anatomical variability of the lateral frontal lobe surface: implication for intersubject variability in language neuroimaging. Neuroimage 24, 504-514.

Julian, J.B., Fedorenko, E., Webster, J., Kanwisher, N., 2012. An algorithmic method for functionally defining regions of interest in the ventral visual pathway. Neuroimage 60, 2357-2364.

Kanai, R., Rees, G., 2011. The structural basis of inter-individual differences in human behaviour and cognition. Nat. Rev. Neurosci. 12, 231-242.

Kanwisher, N., McDermott, J., Chun, M.M., 1997. The fusiform face area: a module in human extrastriate cortex specialized for face perception. J. Neurosci. 17, 4302-4311.

Kawabata Duncan, K.J., Devlin, J.T., 2011. Improving the reliability of functional localizers. Neuroimage 57, 1022-1030.

Kennedy, D.N., Lange, N., Makris, N., Bates, J., Meyer, J., Caviness Jr., V.S., 1998. Gyri of the human neocortex: an MRI-based analysis of volume and variance. Cereb. Cortex 8 372-384.

Knecht, S., Drager, B., Deppe, M., Bobe, L., Lohmann, H., Floel, A., Ringelstein, E.B., Henningsen, H., 2000. Handedness and hemispheric language dominance in healthy humans. Brain 123, 2512-2518.

Koten Jr., J.W., Wood, G., Hagoort, P., Goebel, R., Propping, P., Willmes, K., Boomsma, D.I. 2009. Genetic contribution to variation in cognitive function: an FMRI study in twins. Science 323, 1737-1740.

Lahnakoski, J.M., Glerean, E., Salmi, J., Jaaskelainen, I.P., Sams, M., Hari, R., Nummenmaa, L. 2012. Naturalistic FMRI mapping reveals superior temporal sulcus as the hub for the distributed brain network for social perception. Front. Hum. Neurosci. 6, 233.

Landis, T., Regard, M., Bliestle, A., Kleihues, P., 1988. Prosopagnosia and agnosia for noncanonical views. An autopsied case. Brain 111, 1287-1297.

Liu, J., Harris, A., Kanwisher, N., 2010. Perception of face parts and face configurations: an FMRI study. J. Cogn. Neurosci. 22, 203-211.

Malikovic, A., Amunts, K., Schleicher, A., Mohlberg, H., Eickhoff, S.B., Wilms, M., PalomeroGallagher, N., Armstrong, E., Zilles, K., 2007. Cytoarchitectonic analysis of the human extrastriate cortex in the region of V5/MT +: a probabilistic, stereotaxic map of area hOc5. Cereb. Cortex 17, 562-574.

Mazziotta, J., Toga, A., Evans, A. Fox, P. Lancaster, J. Zilles, K. Woods, R, Paus, T., Simpson, G., Pike, B., Holmes, C., Collins, L., Thompson, P., MacDonald, D., Iacoboni, M., Schormann, T., Amunts, K., Palomero-Gallagher, N., Geyer, S., Parsons, L., Narr, K. Kabani, N., Le Goualher, G., Boomsma, D., Cannon, T., Kawashima, R., Mazoyer, B. 2001. A probabilistic atlas and reference system for the human brain: International Consortium for Brain Mapping (ICBM). Philos. Trans. R. Soc. Lond. B Biol. Sci. 356 1293-1322.

McCarthy, G., Puce, A., Gore, J.C., Allison, T., 1997. Face-specific processing in the human fusiform gyrus. J. Cogn. Neurosci. 9, 605-610.

Meyer, F., 1994. Topographic distance and watershed lines. Signal Process. 38, 113-125.

Mori, S., Oishi, K., Jiang, H., Jiang, L., Li, X., Akhter, K., Hua, K., Faria, A.V., Mahmood, A. Woods, R., Toga, A.W., Pike, G.B., Neto, P.R., Evans, A., Zhang, J., Huang, H., Miller, M.I., van Zijl, P., Mazziotta, J., 2008. Stereotaxic white matter atlas based on diffusion tensor imaging in an ICBM template. Neuroimage 40, 570-582.

Mueller, S., Wang, D., Fox, M.D., Yeo, B.T., Sepulcre, J., Sabuncu, M.R., Shafee, R. Lu, J. Liu, H., 2013. Individual variability in functional connectivity architecture of the human brain. Neuron 77, 586-595.

Nestor, A., Plaut, D.C., Behrmann, M., 2011. Unraveling the distributed neural code of facial identity through spatiotemporal pattern analysis. Proc. Natl. Acad. Sci. U. S. A. 108, 9998-10003.

Nieto-Castanon, A., Fedorenko, E., 2012. Subject-specific functional localizers increase sensitivity and functional resolution of multi-subject analyses. Neuroimage 63, 1646-1669. 
Passingham, R.E., Stephan, K.E., Kotter, R., 2002. The anatomical basis of functional localization in the cortex. Nat. Rev. Neurosci. 3, 606-616.

Phillips, M.L., Young, A.W., Senior, C., Brammer, M., Andrew, C., Calder, A.J., Bullmore, E.T. Perrett, D.I., Rowland, D., Williams, S.C., Gray, J.A., David, A.S., 1997. A specific neural substrate for perceiving facial expressions of disgust. Nature 389, 495-498.

Pinsk, M.A., Arcaro, M., Weiner, K.S., Kalkus, J.F., Inati, S.J., Gross, C.G., Kastner, S., 2009. Neural representations of faces and body parts in macaque and human cortex: a comparative FMRI study. J. Neurophysiol. 101, 2581-2600.

Pitcher, D., Dilks, D.D., Saxe, R.R., Triantafyllou, C., Kanwisher, N., 2011a. Differential selectivity for dynamic versus static information in face-selective cortical regions. Neuroimage 56, 2356-2363.

Pitcher, D., Walsh, V., Duchaine, B., 2011b. The role of the occipital face area in the cortical face perception network. Exp. Brain Res. 209, 481-493.

Polk, T.A., Park, J., Smith, M.R., Park, D.C., 2007. Nature versus nurture in ventral visual cortex: a functional magnetic resonance imaging study of twins. J. Neurosci. 27, 13921-13925.

Polley, D.B., Steinberg, E.E., Merzenich, M.M., 2006. Perceptual learning directs auditory cortical map reorganization through top-down influences. J. Neurosci. 26, 4970-4982.

Puce, A., Allison, T., Bentin, S., Gore, J.C., McCarthy, G., 1998. Temporal cortex activation in humans viewing eye and mouth movements. J. Neurosci. 18, 2188-2199.

Rademacher, J., Morosan, P., Schormann, T., Schleicher, A., Werner, C., Freund, H.J., Zilles, K., 2001. Probabilistic mapping and volume measurement of human primary auditory cortex. Neuroimage 13, 669-683.

Rhodes, G., 1985. Lateralized processes in face recognition. Br. J. Psychol. 76, 249-271.

Rossion, B., Joyce, C.A., Cottrell, G.W., Tarr, M.J., 2003. Early lateralization and orientation tuning for face, word, and object processing in the visual cortex. Neuroimage 20 1609-1624.

Rossion, B., Hanseeuw, B., Dricot, L., 2012. Defining face perception areas in the human brain: a large-scale factorial fMRI face localizer analysis. Brain Cogn. 79, 138-157.

Rottschy, C., Eickhoff, S.B., Schleicher, A., Mohlberg, H., Kujovic, M., Zilles, K., Amunts, K. 2007. Ventral visual cortex in humans: cytoarchitectonic mapping of two extrastriate areas. Hum. Brain Mapp. 28, 1045-1059.

Saxe, R., Brett, M., Kanwisher, N., 2006. Divide and conquer: a defense of functional localizers. Neuroimage 30, 1088-1096.

Saygin, Z.M., Osher, D.E., Koldewyn, K., Reynolds, G., Gabrieli, J.D., Saxe, R.R., 2011. Anatomical connectivity patterns predict face selectivity in the fusiform gyrus. Nat Neurosci. 15, 321-327.

Scheperjans, F., Eickhoff, S.B., Homke, L., Mohlberg, H., Hermann, K., Amunts, K., Zilles, K, 2007. Probabilistic maps, morphometry, and variability of cytoarchitectonic areas in the human superior parietal cortex. Cereb. Cortex 18, 2141-2157.

Schiltz, C., Rossion, B., 2006. Faces are represented holistically in the human occipitotemporal cortex. Neuroimage 32, 1385-1394.

Shattuck, D.W., Mirza, M., Adisetiyo, V., Hojatkashani, C., Salamon, G., Narr, K.L., Poldrack, R.A., Bilder, R.M., Toga, A.W., 2008. Construction of a 3D probabilistic atlas of human cortical structures. Neuroimage 39, 1064-1080.

Song, Y., Hu, S., Li, X., Li, W., Liu, J., 2010. The role of top-down task context in learning to perceive objects. J. Neurosci. 30, 9869-9876.

Song, Y., Tian, M., Liu, J., 2012. Top-down processing of symbolic meanings modulates the visual word form area. J. Neurosci. 32, 12277-12283.
Sugiura, M., Friston, K.J., Willmes, K., Shah, N.J., Zilles, K., Fink, G.R., 2007. Analysis of intersubject variability in activation: an application to the incidental episodic retrieval during recognition test. Hum. Brain Mapp. 28, 49-58.

Susilo, T., Germine, L., Duchaine, B., 2013. Face recognition ability matures late: evidence from individual differences in young adults. J. Exp. Psychol. Hum. Percept. Perform. 39, 1212-1217.

Swallow, K.M., Braver, T.S., Snyder, A.Z., Speer, N.K., Zacks, J.M., 2003. Reliability of functional localization using fMRI. Neuroimage 20, 1561-1577.

Tahmasebi, A.M., Artiges, E., Banaschewski, T., Barker, G.J., Bruehl, R., Buchel, C., Conrod, P.J., Flor, H., Garavan, H., Gallinat, J., Heinz, A., Ittermann, B., Loth, E., Mareckova, K., Martinot, J.L., Poline, J.B., Rietschel, M., Smolka, M.N., Strohle, A., Schumann, G., Paus, T., 2012. Creating probabilistic maps of the face network in the adolescent brain: a multicentre functional MRI study. Hum. Brain Mapp. 33, 938-957.

Thompson, P.M., Schwartz, C., Lin, R.T., Khan, A.A., Toga, A.W., 1996. Three-dimensional statistical analysis of sulcal variability in the human brain. J. Neurosci. 16, 4261-4274.

Thompson, P.M., Moussai, J., Zohoori, S., Goldkorn, A., Khan, A.A., Mega, M.S., Small, G.W., Cummings, J.L., Toga, A.W., 1998. Cortical variability and asymmetry in normal aging and Alzheimer's disease. Cereb. Cortex 8, 492-509.

Toga, A.W., Thompson, P.M., Mori, S., Amunts, K., Zilles, K., 2006. Towards multimodal atlases of the human brain. Nat. Rev. Neurosci. 7, 952-966.

Van Essen, D.C., Dierker, D.L., 2007. Surface-based and probabilistic atlases of primate cerebral cortex. Neuron 56, 209-225

Van Horn, J.D., Grafton, S.T., Miller, M.B., 2008. Individual variability in brain activity: a nuisance or an opportunity? Brain Imaging Behav. 2, 327-334.

Weiner, K.S., Grill-Spector, K., 2010. Sparsely-distributed organization of face and limb activations in human ventral temporal cortex. Neuroimage 52, 1559-1573.

Weiner, K.S., Grill-Spector, K., 2013. Neural representations of faces and limbs neighbor in human high-level visual cortex: evidence for a new organization principle. Psychol. Res. 77 (1), 74-97.

Xiong, J., Rao, S., Jerabek, P., Zamarripa, F., Woldorff, M., Lancaster, J., Fox, P.T., 2000. Intersubject variability in cortical activations during a complex language task. Neuroimage 12, 326-339.

Yovel, G., Kanwisher, N., 2005. The neural basis of the behavioral face-inversion effect. Curr. Biol. 15, 2256-2262.

Yovel, G., Levy, J., Grabowecky, M., Paller, K.A., 2003. Neural correlates of the left-visualfield superiority in face perception appear at multiple stages of face processing. J. Cogn. Neurosci. 15, 462-474.

Zhen, Z., Fang, H., Liu, J., 2013. The hierarchical brain network for face recognition. PLoS One 8, e59886.

Zhu, Q., Zhang, J., Luo, Y.L., Dilks, D.D., Liu, J., 2011. Resting-state neural activity across face-selective cortical regions is behaviorally relevant. J. Neurosci. 31, 10323-10330.

Zilles, K., Amunts, K., 2010. Centenary of Brodmann's map - conception and fate. Nat. Rev. Neurosci. 11, 139-145.

Zilles, K., Amunts, K., 2013. Individual variability is not noise. Trends Cogn. Sci. 17, 153-155.

Zilles, K., Palomero-Gallagher, N., Amunts, K., 2013. Development of cortical folding during evolution and ontogeny. Trends Neurosci. 36, 275-284. 